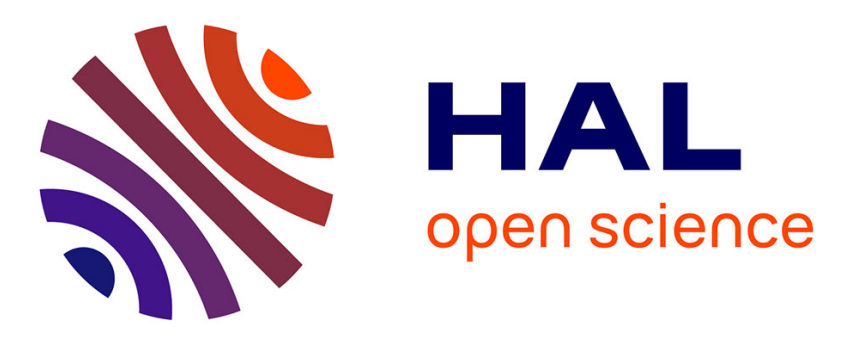

\title{
Energy Partitioning Control in the PITM Hybrid RANS/LES Method for the Simulation of Turbulent Flows
}

\author{
Bruno Chaouat, Roland Schiestel
}

\section{- To cite this version:}

Bruno Chaouat, Roland Schiestel. Energy Partitioning Control in the PITM Hybrid RANS/LES Method for the Simulation of Turbulent Flows. Flow, Turbulence and Combustion, In press, 10.1007/s10494-021-00259-w . hal-03229080

\section{HAL Id: hal-03229080 \\ https://hal.science/hal-03229080}

Submitted on 18 May 2021

HAL is a multi-disciplinary open access archive for the deposit and dissemination of scientific research documents, whether they are published or not. The documents may come from teaching and research institutions in France or abroad, or from public or private research centers.
L'archive ouverte pluridisciplinaire HAL, est destinée au dépôt et à la diffusion de documents scientifiques de niveau recherche, publiés ou non, émanant des établissements d'enseignement et de recherche français ou étrangers, des laboratoires publics ou privés. 


\title{
Energy partitioning control in the PITM hybrid RANS/LES method for the simulation of turbulent flows
}

\author{
Bruno Chaouat ONERA, Université Paris-Saclay, 92322 Châtillon, France \\ Roland Schiestel IRPHE/CNRS, 13384 Marseille, France
}

March 31, 2021

\begin{abstract}
The partially integrated transport modeling (PITM) method first introduced in Ref. [R. Schiestel and A. Dejoan, "Towards a new partially integrated transport model for coarse grid and unsteady turbulent flow simulations ", Theor. Comput. Fluid Dyn. 18, 443 (2005)] and in Ref. [B. Chaouat and R. Schiestel, "A new partially integrated transport model for subgrid-scale stresses and dissipation rate for turbulent developing flows ", Phys. Fluids 17, 065106 (2005)] provides a continuous approach for hybrid RANS-LES simulations. Inspired from the multiple scale approach, the basis of the development of the method is the spectral space in quasi-homogeneous turbulence. The PITM method embodies a partitioning control function that monitors the ratio of subfilter energy to total turbulent energy by reference to the cutoff wavenumber location. How this procedure behaves in inhomogeneous flows is an important question. The present paper demonstrates that the same control function can be used both in homogeneous and in non-homogeneous flows as well. Further on, an analysis of the effect of anisotropic filters generally used for wall flows is conducted for computing the equivalent cutoff wavenumber suitable for determining the subfilter energy of the spectrum and see how it interferes with control function. Illustrations in the turbulent plane channel flow are given that confirm the efficiency of the procedure and DNS data have been used to support and supplement the discussion.
\end{abstract}




\section{Introduction}

Both Reynolds Averaged Navier-Stokes equations (RANS) and Large Eddy Simulations (LES) often used in Computational Fluid Dynamic (CFD) have their advantages and their drawbacks for the simulation of turbulent flows $[1,2,3]$. On the one hand, the Reynolds Averaged Navier-Stokes (RANS) methodology based on a statistical averaging or in practice a long-time averaging is often used in industry for tackling engineering flows encountered in aeronautics applications. This method performs well for turbulent flows in relatively simple geometries with reasonable computational costs provided the mean flow quantities vary slowly in time and space. On the other hand, highly resolved Large-Eddy Simulation (LES) which consists in modeling the more universal small scales while the large scales motions are explicitly calculated is a promising route for reproducing accurately the details of unsteady flows but, up to now, it still remains not affordable for industrial applications involving large computational domains, even with the rapid increase of super-computer power $[1,2,3]$. Another issue in industrial LES is the need for a very long computational time, especially when low-frequency phenomena are involved, for instance in situations with flow separation. Hybrid RANS/LES methods that combine both advantages of RANS and LES methods have been developed in the past two decades in this context for simulating practical turbulent flows and produced fair results and reasonable computational resources are required $[2,3,4,5,6]$. Among these methods, the main schools of modeling that can be retained as representative are the so-called Very Large Eddy Simulation (VLES) [7], the Detached Eddy Simulation (DES) $[8,9]$, the Partially Integrated Transport Modeling (PITM) method [10, 11], the Partially Averaged Navier-Stokes (PANS) method [12, 13, 14], the Scale Adaptive Simulation (SAS) [15, 16]. Among these different methods, the PITM method gains its particular interest in its ability to bridge the RANS and LES approaches with seamless coupling and thus it avoids the overwhelming problems caused by the so-called gray zone that are usually posed by zonal hybrid RANS/LES methods. In all these hybrid methods, the subfilter part of the turbulence energy is usually substantial and for this reason turbulence transport models are appropriate and justified, while they would not be necessary in fine grid LES.

Continuous hybrid models for turbulence simulation like the PITM [10, 11] approach provide a 
seamless passage from LES behavior to RANS type behavior in respect to the grid-size of the mesh and turbulence length-scale. The PITM method developed by Schiestel and Dejoan [10] for two-equation subfilter scale (SFS) energy models and Chaouat and Schiestel [11] for subfilter scale stress transport models based on second-moment closure (SMC) is funded originally on the spectral equations for quasihomogeneous turbulence $[17,18,19]$ and from using a partial integration of an analytical Von Kármán like energy spectrum to establish a link between the subfilter energy and the grid [20]. The energy splitting technique used in PITM for turbulent flows is of general character and has been recently extended to passive scalar fields [21]. If considering the hypothesis of tangent homogeneous space [22], it becomes then possible to deal with non-homogeneous flows with a good approximation for practice. An important parameter is given by the ratio of the subfilter energy $\left\langle k^{(s)}\right\rangle$ to the total energy of turbulence $k$ denoted as $r=\left\langle k^{(s)}\right\rangle / k$. This parameter was introduced in the PITM [10, 11] and also in the PANS $[12,13,14]$ methods for controlling the portion of turbulence energy modeled in the subgrid scale level although these two methods are based on different argument involving either the spectral space or the physical space [3]. In the PITM framework, this partial energy parameter is connected to the cutoff wavenumber in order to control the fraction of energy of the simulation in relation to the calculation grid density and the turbulence length-scale. It is thus dynamic in time and space for the PITM [11, 23] while it is assumed to be a constant in the original PANS method in practice for CFD applications [14]. The appropriateness of the grid refinement is obviously disregarded and consequently, the consistency between the mesh and the subfilter energy is no longer automatically fulfilled as it is in PITM [24]. This is in fact, one of the main differences between the resulting final equations obtained in PANS compared to PITM, as shown in the review paper of Chaouat [3]. Note that a new formulation of the PANS method was subsequently devised using partial integration of the complete Von Kármán energy spectrum $E(\kappa)$ as introduced earlier in PITM to correct this drawback [25, 26, 27]. An additional development has been made on the PANS model to accurately reproduce the boundary layer and the details of the wall flow structure attributed to hairpin vortices and ejection/sweep mechanisms in channel flows but by loosing seamless coupling in sacrifice [28, 29].

By way of illustration, one can mention the turbulent flow over periodic hills that constitutes a challenging test case that embodies turbulence mechanisms associated with separation, recirculation, 
reattachment and acceleration. This flow was performed on coarse grids by Chaouat [30], Chaouat and Schiestel [23] using the PITM at the Reynolds number $R_{e}=10595$ and 37000, respectively, as well as Razi et al. [24] using the PANS at both Reynolds numbers, while returning satisfying results. More recently, Heinz et al. [31, 32, 33] have taken up in its principle the PITM method concerning the dependence of the coefficient $C_{\epsilon_{2}}^{(s)}$ in the dissipation-rate equation $\epsilon$ to derive a variant model called Continuous Eddy Simulation (CES) based of the transport equation for the turbulence frequency $\omega=\epsilon / k$. This method was used to simulate the two-dimensional periodic hill flow at the Reynolds number 37000 [5]. Note that Jakirlić and Maduta [34] also performed this flow at Re $=10595$ using the IS-RSM model in line with the SAS.

Two steps in PITM are identified in the control mechanism to monitor the energy ratio in relation to the filter width, or in practice the grid size of the mesh. The first step determines the connection between the model parameter and the energy ratio $r$ by specifying the function $C_{\epsilon_{2}}^{(s)}$. The second step gives a connection between the ratio $r=\left\langle k^{(s)}\right\rangle / k$ and the cutoff wavenumber or equivalently, the filter width. We will demonstrate in the following that the first step obtained in homogeneous turbulence remains still valid in all cases, including homogeneous and non-homogeneous flows. In order to get an efficient control, both steps are necessary. Indeed, when trying to interpret physically the meaning of the parameter ratio $r=\left\langle k^{(s)}\right\rangle / k$, one easily realize that there are at least two different reasons for a variation in its value. The first reason can be attributed to a change in the filter width compared to the turbulence macroscale [35] and the second reason can be due to a change in the spectral distribution corresponding to a departure from equilibrium [20]. These two different cases are sketched in Figs. 1 and 2 , illustrating the evolving energy spectrum with the same decrease in $r$. As a consequence, it appears also mandatory to provide a link between the cutoff location and the subfilter energy level in order to get control at the length scale level. As mentioned above, this fact was recognized in the very early versions of the PITM method $[10,11]$ that introduces a direct link between the ratio $r$ and the filter width or the cutoff wavenumber $\kappa_{c}$ that is usually equal to the grid size. An additional important point which interferes with the function $C_{\epsilon}^{(s)}(r)$ is the concern of the necessary use of anisotropic filters in non-homogeneous flows such as turbulent wall flows. Indeed, the filter anisotropy may significantly alter the determination of the equivalent cutoff wavenumber and consequently the function $C_{\epsilon_{2}}^{(s)}\left[r\left(\kappa_{c}\right)\right]$. 


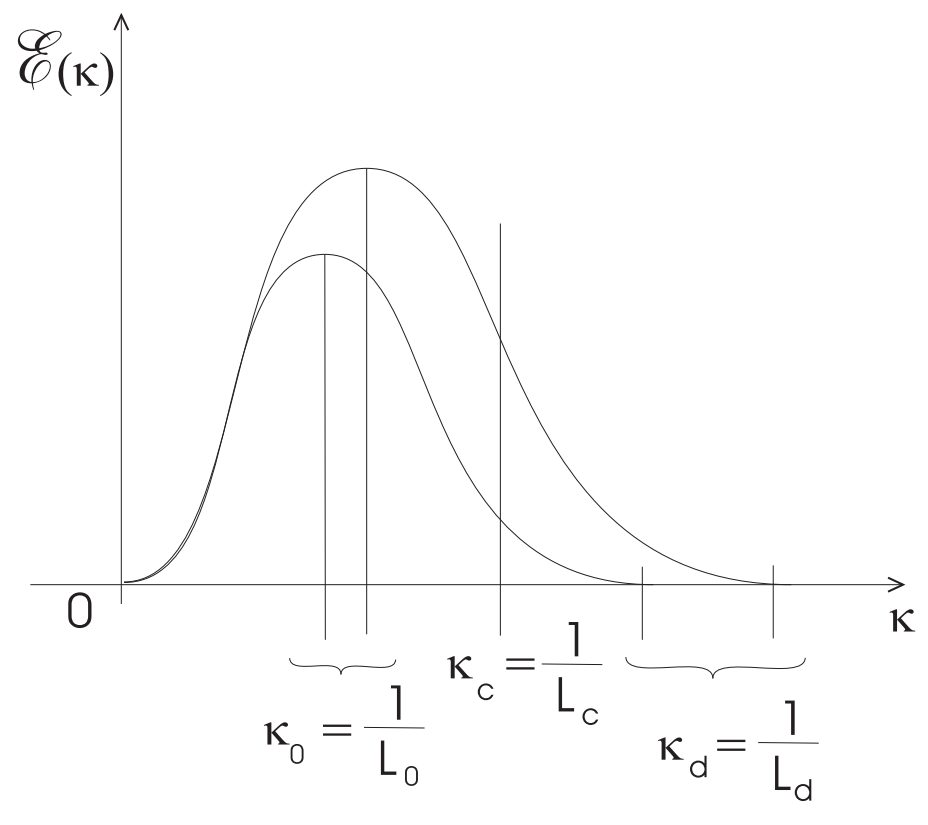

Figure 1: Variation of $r$ due to self-similar near equilibrium decay $L_{0}$ may vary, $L_{c}$ fixed and $L_{d}$ may vary

As it was pointed out above, the PITM method was developed in a spectral framework with the hypothesis of homogeneous turbulence or the hypothesis of locally homogeneous turbulence [22]. In this approach, the turbulence diffusion terms in the dissipation-rate equation were introduced subsequently as an empirical addition. Obviously, the control function $R(r)=r$ in the dissipation-rate transport equation represented as an adjustable varying parameter in the source term

$$
C_{\epsilon_{2}}^{(s)}=C_{\epsilon_{1}}^{(s)}+R(r)\left(C_{\epsilon_{2}}-C_{\epsilon_{1}}\right)
$$

where $C_{\epsilon_{1}}$ and $C_{\epsilon_{2}}$ are the constant coefficients used in the RANS dissipation-rate equation, has been kept for the simulation of non-homogeneous flows. The linear dependence of the function $R(r)$ has been questioned recently by Heinz et al. [31, 32, 33] so that it should be necessary to clarify the situation. We will demonstrate in this work that the value $R=r$ found in locally homogeneous flows in the framework of the PITM method still remains valid in non-homogeneous turbulent flows. 


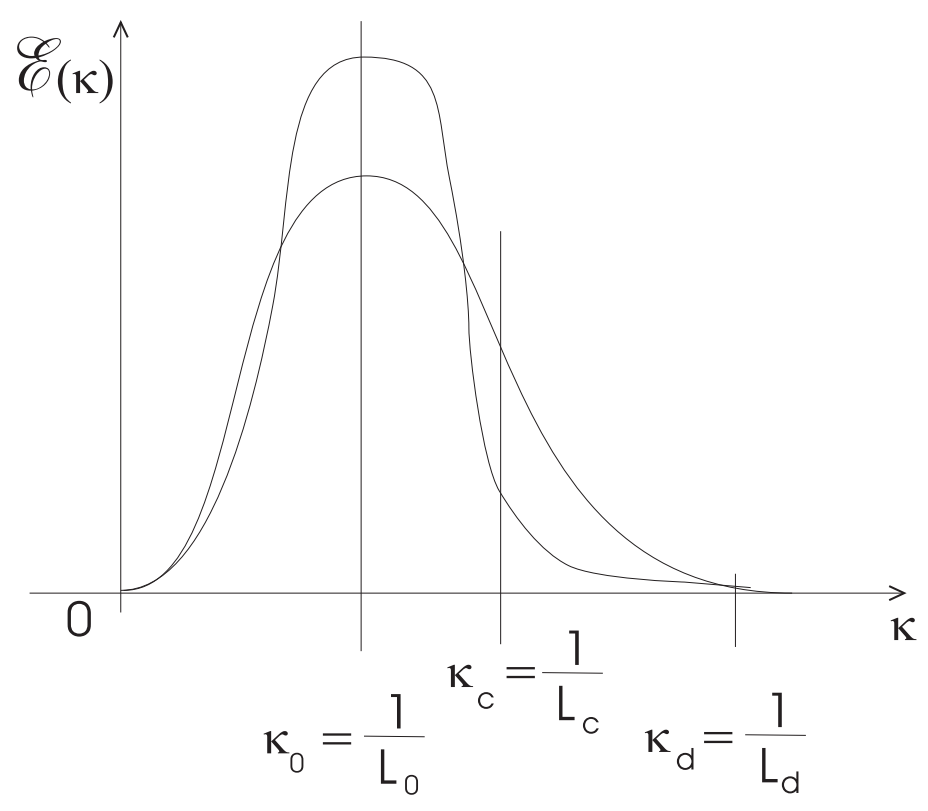

Figure 2: Variation of $r$ due to strong departure from equilibrium $L_{c}$ fixed and $L_{0}, L_{d}$ almost const.

\section{Mathematical framework}

\subsection{Averaging and filtering processes}

Turbulent flow of a viscous incompressible flow is considered. In RANS methodology, each variable $\phi$ can be decomposed into a statistical part $\langle\phi\rangle$ and a fluctuating part $\phi^{\prime}$ such as $\phi=\langle\phi\rangle+\phi^{\prime}$ whereas in large eddy simulation, the variable $\phi$ is decomposed into a large scale (or resolved part) $\bar{\phi}$ and a subfilter-scale fluctuating part $\phi^{>}$or modeled part such that $\phi=\bar{\phi}+\phi^{>}$. The instantaneous fluctuation $\phi^{\prime}$ contains in fact the large scale fluctuating part $\phi^{<}$and the small scale fluctuating part $\phi^{>}$such that $\phi^{\prime}=\phi^{<}+\phi^{>}$. So that the instantaneous variable $\phi$ can be then rewritten as the sum of the mean statistical part $\langle\phi\rangle$, the large scale fluctuating part $\phi^{<}=\bar{\phi}-\langle\phi\rangle$, and the small scale fluctuating part $\phi^{>}$. The filtered variable $\bar{\phi}$ is defined by the filtering operation as the convolution with a filter $G$ in physical space $\bar{\phi}=G * \phi$ that leads to the computation of a variable convolution integral [35, 36]. As known, the properties of the filtering process are different from those of the statistical averaging process. In particular, $\overline{\bar{\phi}} \neq \bar{\phi}$, 
$\left\langle\phi^{<}\right\rangle=-\left\langle\phi^{>}\right\rangle \neq 0$. As a result of interest, one note also that $\langle\bar{\phi}\rangle=\langle\phi\rangle-\left\langle\phi^{>}\right\rangle \neq\langle\phi\rangle$ so that there is no direct connection between the averaged field in a statistical sense and the filtered field in LES. But, all these difficulties disappear in an homogeneous turbulent field if the spectral cutoff filter is used [22, 35]. In this case, the large scale fluctuations (resolved scales) and the fine scale fluctuations (modeled scales) are formally defined from the physical meaning of the Fourier transform of the fluctuating quantities $\phi^{\prime}$ using the cutoff wavenumber $\kappa_{c}$ as the lower bound of the integration interval. In the physical space, the large scale $\phi^{<}$and the fine scale $\phi^{>}$are then defined from the well-known Fourier transforms

$$
\begin{aligned}
& \phi^{<}=\int_{|\kappa| \leq \kappa_{c}} \widehat{\phi^{\prime}}(\boldsymbol{X}, \boldsymbol{\kappa}) \exp (j \boldsymbol{\kappa} \boldsymbol{\xi}) d \boldsymbol{\kappa} \\
& \phi^{>}=\int_{|\kappa| \geq \kappa_{c}} \widehat{\phi^{\prime}}(\boldsymbol{X}, \boldsymbol{\kappa}) \exp (j \boldsymbol{\kappa} \boldsymbol{\xi}) d \boldsymbol{\kappa}
\end{aligned}
$$

where $\kappa_{c}$ denotes the cutoff wave number defined as $\kappa_{c}=\pi / \Delta$ accounting for the filter width $\Delta$ which may be larger than the grid-size of the mesh. In the general case, it is useful to refer to the tangent homogeneous space at a point of a non-homogeneous flow field assuming Taylor series expansion in space for the mean velocity field in the derivation of transport equations of turbulence because the large scale and small scale fluctuations are uncorrelated $\left\langle\phi^{>} \phi^{<}\right\rangle=0$. Moreover, it appears that $\overline{\langle\phi\rangle}=\langle\bar{\phi}\rangle=\langle\phi\rangle$ [35]. In general practice however, it is assumed that $\langle\bar{\phi}\rangle \approx\langle\phi\rangle$ only if the variation the filter function is not too progressive (sufficiently sharp).

\subsection{Structure of the turbulence transport equations}

Recalling main results obtained in previous publications [10, 11], the dissipation-rate equation in the PITM method was thus derived for homogeneous flows as

$$
\frac{\partial \varepsilon^{(s)}}{\partial t}=C_{\epsilon_{1}}^{(s)} \frac{P^{(s)} \varepsilon^{(s)}}{k^{(s)}}-C_{\epsilon_{2}}^{(s)} \frac{\left(\varepsilon^{(s)}\right)^{2}}{k^{(s)}}
$$

The function $C_{\epsilon_{2}}^{(s)}(r)$ described above has been established as a linear function

$$
C_{\epsilon_{2}}^{(s)}=C_{\epsilon_{1}}^{(s)}+r\left(C_{\epsilon_{2}}-C_{\epsilon_{1}}\right)
$$

where

$$
C_{\epsilon_{1}}^{(s)}=C_{\epsilon_{1}}
$$


with $0 \leqslant r \leqslant 1$, and $r=\left\langle k^{(s)}\right\rangle / k$. We will show in the next section that this result remains valid for non-homogeneous flows. In a second step, the energy ratio $r$ was then connected to the cutoff value by using spectral integration of a Von Kármán like spectrum [20]

$$
\frac{\left\langle k^{(s)}\right\rangle}{k}=\frac{1}{\left(1+\beta \eta_{c}^{3}\right)^{2 / 9}}
$$

to obtain the result $[20]$

$$
C_{\epsilon_{2}}^{(s)}=C_{\epsilon_{1}}+\frac{C_{\epsilon_{2}}-C_{\epsilon_{1}}}{\left(1+\beta \eta_{c}^{3}\right)^{2 / 9}}
$$

with

$$
\eta_{c}=\kappa_{c} L=\frac{\pi}{\Delta} \frac{k^{3 / 2}}{\varepsilon}=\frac{\pi L}{\Delta}
$$

where $\beta=\left[2 /\left(3 C_{\kappa}\right)\right]^{9 / 2}$ and $C_{\kappa} \approx 1.5$ is the Kolmogorov constant. The limiting values $r=1$ and $r=0$ correspond to the full RANS modeling and DNS, respectively. In the limiting condition when $\eta_{c}$ goes to infinity, then

$$
\lim _{\eta_{c} \rightarrow \infty} \frac{1}{\left(1+\beta \eta_{c}^{3}\right)^{2 / 9}}=\frac{1}{\beta^{2 / 9}} \eta_{c}^{-2 / 3}
$$

The relation (8) can be meaningful only if the $r$ dependence in (5) and (6) of the first step is correct. This point is thus relevant to the first step described above and it deserves specific interest. This question was addressed by Heinz et al. [31, 32, 33] and will be discussed in the present paper in the following section with the aim of confirming the validity of relations (5) and (6) in all cases. The demonstration is based on a more detailed account of the statistically averaged subfilter equations, compared to the non averaged ones. Indeed, the equations solved in the subfilter model are applied to the unsteady large scale motions that are already a part of the fluctuating turbulent flow field. The statistical mean counterparts of these equations have also been largely studied by Chaouat and Schiestel [22]. These equations are similar in their general form but the physical interpretation of the various terms gives important informations that are the key for the demonstration of the next section. As the reasoning will rely upon the subfilter scale transport equations as developed by Germano [37] and also by Chaouat and Schiestel [22]. The unsteady transport equation for the subfilter energy $k^{(s)}=\tau\left(u_{j}, u_{j}\right) / 2$ reads 
$[22,37]$

$$
\begin{aligned}
& \frac{\partial k^{(s)}}{\partial t}+\left\langle u_{j}\right\rangle \frac{\partial k^{(s)}}{\partial x_{j}}=-\frac{\partial}{\partial x_{j}}\left[\frac{1}{2} \tau\left(u_{i}, u_{i}, u_{j}\right)+u_{j}^{<} k^{(s)}\right]+\nu \frac{\partial^{2} k^{(s)}}{\partial x_{j} \partial x_{j}} \\
& -\frac{1}{\rho} \frac{\partial \tau\left(p, u_{i}\right)}{\partial x_{i}}-\nu \tau\left(\frac{\partial u_{i}}{\partial x_{j}}, \frac{\partial u_{i}}{\partial x_{j}}\right)-\tau\left(u_{i}, u_{j}\right) \frac{\partial\left\langle u_{i}\right\rangle}{\partial x_{j}}-\tau\left(u_{i}, u_{j}\right) \frac{\partial u_{i}^{<}}{\partial x_{j}}
\end{aligned}
$$

where the functions $\tau(f, g)$ and $\tau(f, g, h)$ are defined in Appendix A. Using derivative operators defined in Appendix B, it is then possible to rewrite this equation in a more compact form as

$$
\begin{gathered}
\frac{\mathcal{D} k^{(s)}}{\mathcal{D} t}=\frac{\partial k^{(s)}}{\partial t}+\bar{u}_{j} \frac{\partial k^{(s)}}{\partial x_{j}}=-\frac{\partial}{\partial x_{j}}\left[\frac{1}{2} \tau\left(u_{i}, u_{i}, u_{j}\right)\right]+\nu \frac{\partial^{2} k^{(s)}}{\partial x_{j} \partial x_{j}} \\
-\frac{1}{\rho} \frac{\partial \tau\left(p, u_{i}\right)}{\partial x_{i}}-\nu \tau\left(\frac{\partial u_{i}}{\partial x_{j}}, \frac{\partial u_{i}}{\partial x_{j}}\right)-\tau\left(u_{i}, u_{j}\right) \frac{\partial\left\langle u_{i}\right\rangle}{\partial x_{j}}-\tau\left(u_{i}, u_{j}\right) \frac{\partial u_{i}^{<}}{\partial x_{j}}
\end{gathered}
$$

Although, it is the unsteady equations that are solved numerically in the simulation, the terms of these equations have non-zero mean values. So, their averaged version deserve attention because they have to be verified also. The averaging in a statistical sense of Eq. (11) reads

$$
\frac{D\left\langle k^{(s)}\right\rangle}{D t}=\frac{\partial\left\langle k^{(s)}\right\rangle}{\partial t}+\left\langle u_{j}\right\rangle \frac{\partial\left\langle k^{(s)}\right\rangle}{\partial x_{j}}=P^{(2)}+F^{(1)}-F^{(2)}+J^{(2)}
$$

The terms in the RHS, where superscript (1) pertains to the resolved zone and superscript (2) pertains to the subfilter zone, are defined as :

$$
\begin{gathered}
P^{(2)}=-\left\langle\tau_{i j}^{(s)}\right\rangle \frac{\partial\left\langle u_{i}\right\rangle}{\partial x_{j}} \\
F^{(1)}=-\left\langle\tau_{i j}^{(s)} \frac{\partial u_{i}^{<}}{\partial x_{j}}\right\rangle \\
F^{(2)}=\nu\left\langle\tau\left(\frac{\partial u_{i}}{\partial x_{j}}, \frac{\partial u_{i}}{\partial x_{j}}\right)\right\rangle=\nu\left\langle\frac{\partial u_{i}^{>}}{\partial x_{j}} \frac{\partial u_{i}^{>}}{\partial x_{j}}\right\rangle \approx \varepsilon \\
J^{(2)}=-\frac{\partial}{\partial x_{j}}\left[\frac{1}{2}\left\langle u_{i}^{>} u_{i}^{>} u_{j}^{>}\right\rangle+\left\langle u_{j}^{<} k^{(s)}\right\rangle\right]-\frac{1}{\rho} \frac{\partial}{\partial x_{i}}\left\langle p^{>} u_{i}^{>}\right\rangle+\nu \frac{\partial^{2}\left\langle k^{(s)}\right\rangle}{\partial x_{j} \partial x_{j}}
\end{gathered}
$$

The diffusion term $J^{(2)}$ contains both a diffusion due to the small scales $J^{(2), s}$ and a diffusion due to the large resolved scales $J^{(2), r}$ in addition to the molecular term $J_{\nu}^{(2)}$. This is clear when rewriting $J^{(2)}$ as

$$
J^{(2)}=\underbrace{-\frac{\partial}{\partial x_{j}}\left\langle u_{j}^{<} k^{(s)}\right\rangle}_{J^{(2), r}} \underbrace{-\frac{\partial}{\partial x_{j}} \frac{1}{2}\left\langle u_{j}^{>} u_{i}^{>} u_{i}^{>}\right\rangle-\frac{1}{\rho} \frac{\partial}{\partial x_{i}}\left\langle p^{>} u_{i}^{>}\right\rangle}_{J^{(2), s}}+\underbrace{\nu \frac{\partial^{2}\left\langle k^{(s)}\right\rangle}{\partial x_{j} \partial x_{j}}}_{J_{\nu}^{(2)}}
$$


The production term in the subfilter kinetic energy equation (12) is defined as

$$
P^{(s)}=-\tau_{i j}^{(s)} \frac{\partial \bar{u}_{i}}{\partial x_{j}}=-\tau_{i j}^{(s)} \frac{\partial\left\langle u_{i}\right\rangle}{\partial x_{j}}-\tau_{i j}^{(s)} \frac{\partial u_{i}^{<}}{\partial x_{j}}
$$

and its mean value then equal to

$$
\left\langle P^{(s)}\right\rangle=-\left\langle\tau_{i j}^{(s)} \frac{\partial \bar{u}_{i}}{\partial x_{j}}\right\rangle=-\left\langle\tau_{i j}^{(s)}\right\rangle \frac{\partial\left\langle u_{i}\right\rangle}{\partial x_{j}}-\left\langle\tau_{i j}^{(s)} \frac{\partial u_{i}^{<}}{\partial x_{j}}\right\rangle
$$

that is to say

$$
\left\langle P^{(s)}\right\rangle=-\left(P^{(2)}+F^{(1)}\right)
$$

The production $P^{(s)}$ is an unsteady fluctuating production term, while $P^{(2)}=-\left\langle\tau_{i j}^{(s)}\right\rangle \partial\left\langle u_{i}\right\rangle / \partial x_{j}$ is the statistical production related to mean flow and $F^{(1)}=-\left\langle\tau_{i j}^{(s)} \partial u_{i}^{<} / \partial x_{j}\right\rangle$ denotes the mean spectral cascade flux through the cutoff. We recall that $\bar{u}_{j}=\left\langle u_{j}\right\rangle+u_{j}^{<}$. From these equations, it is worth emphasizing in particular that the production of energy from the mean flow $\left\langle P^{(s)}\right\rangle$ in statistical models is replaced in the large eddies simulation by the production $P^{(s)}$ from the filtered motion, which is thus a fluctuating quantity. It embodies a contribution from the mean flow and a contribution from the large scales as shown in equations (19) and (21) as well. Considering an eddy viscosity closure, would lead to the approximation

$$
\tau_{i j}^{(s)}=-\nu_{t}^{(s)}\left(\frac{\partial \bar{u}_{i}}{\partial x_{j}}+\frac{\partial \bar{u}_{j}}{\partial x_{i}}\right)+\frac{2}{3} k^{(s)} \delta_{i j}
$$

and then

$$
\left\langle P^{(s)}\right\rangle=\left\langle\nu_{t}^{(s)}\left(\frac{\partial \bar{u}_{i}}{\partial x_{j}}+\frac{\partial \bar{u}_{j}}{\partial x_{i}}\right) \frac{\partial \bar{u}_{i}}{\partial x_{j}}\right\rangle=2\left\langle\nu_{t}^{(s)} \bar{S}_{i j} \bar{S}_{i j}\right\rangle
$$

with

$$
S_{i j}=\frac{1}{2}\left(\frac{\partial u_{i}}{\partial x_{j}}+\frac{\partial u_{j}}{\partial x_{i}}\right)
$$

As already mentioned above, another important remark applies to the turbulence diffusion terms in the turbulence subfilter transport equations. In the statistical equations, the diffusion term for full quantities (all the spectrum is integrated) like $k$ is approximated by a gradient diffusion term using scalar or tensorial diffusivities of order $C_{k} k^{2} / \varepsilon$. When solving the unsteady subfilter quantities like $k^{(s)}$ the diffusivity will be of order $C_{k} k^{(s)^{2}} / \epsilon^{(s)}$ being a fluctuating quantity. But the diffusion for the mean value $k^{(s)}$ is not just the mean value of the diffusion, it is different, because of unsteadiness as mentioned in the previous section and in Refs. [22, 38]. In the case where the variation of the filter function is 
sufficiently sharp [22, 36, 35], we get $\tau_{i j}^{(s)} \approx \overline{u_{i}^{>} u_{j}^{>}}$, and $\tau_{i j}^{(r)} \approx \overline{u_{i}^{<} u_{j}^{<}}$implying that $k^{(s)} \approx \overline{u_{i}^{>} u_{i}^{>}} / 2$ and $k^{(r)} \approx \overline{u_{i}^{<} u_{i}^{<}} / 2$ and therefore $k \approx\left\langle k^{(s+r)}\right\rangle$ where $k^{(s+r)}=k^{(s)}+k^{(r)}$ so that $k \approx\left[\left\langle u_{i}^{<} u_{i}^{<}\right\rangle+\left\langle u_{i}^{>} u_{i}^{>}\right\rangle\right] / 2$. Moreover, the particle derivatives verify

$$
\overline{\frac{d}{d t} \frac{u_{i}^{>} u_{i}^{>}}{2}}=\frac{\partial k^{(s)}}{\partial t}+\left\langle u_{j}\right\rangle \frac{\partial k^{(s)}}{\partial x_{j}}+u_{j}^{<} \frac{\partial k^{(s)}}{\partial x_{j}}+\frac{\partial \overline{u_{j}^{>} u_{i}^{>} u_{i}^{>}}}{\partial x_{j}}
$$

Taking the averaging of Eq. (25) allows to get

$$
\left\langle\frac{d}{d t} \frac{u_{i}^{>} u_{i}^{>}}{2}\right\rangle=\underbrace{\frac{\partial\left\langle k^{(s)}\right\rangle}{\partial t}+\left\langle u_{j}\right\rangle \frac{\partial\left\langle k^{(s)}\right\rangle}{\partial x_{j}}}_{\frac{D\left\langle k^{(s)}\right\rangle}{D t}}+\underbrace{\frac{\partial}{\partial x_{j}}\left\langle u_{j}^{<} k^{(s)}\right\rangle}_{\frac{\partial}{\partial x_{j}} \Phi_{j}^{(r)}}+\underbrace{\frac{\partial}{\partial x_{j}}\left\langle u_{j}^{>} u_{i}^{>} u_{i}^{>}\right\rangle}_{\frac{\partial}{\partial x_{j}} \Phi_{j}^{(s)}}
$$

So, $\Phi_{j}^{(r)} \approx\left\langle u_{j}^{<} k^{(s)}\right\rangle$ is involving the macrofluctuations velocities. This is reflecting the fact that even if $\left\langle k^{(s)}\right\rangle$ is a sub-filter quantity, all the turbulent scales (small and large) contribute to turbulent diffusion of the mean value. Applying (152) with $\phi=k^{(s)}$, one can obtain

$$
\left\langle\frac{\mathcal{D} k^{(s)}}{\mathcal{D} t}\right\rangle=\frac{D\left\langle k^{(s)}\right\rangle}{D t}+\frac{\partial\left\langle u_{j}^{<} k^{(s)}\right\rangle}{\partial x_{j}}
$$

and equivalently for epsilon

$$
\left\langle\frac{\mathcal{D} \varepsilon^{(s)}}{\mathcal{D} t}\right\rangle=\frac{D\left\langle\varepsilon^{(s)}\right\rangle}{D t}+\frac{\partial\left\langle u_{j}^{<} \varepsilon^{(s)}\right\rangle}{\partial x_{j}}
$$

The fluctuating inertial term in the particular derivative which is entirely resolved in the large eddies simulation, is then interpreted as a turbulent diffusion term in the statistical context. This term is fully resolved in the simulation, but for the need of the following argumentation, an approximated form is given Appendix $\mathrm{C}$ to get an estimate. These previous remarks will be essential in the following developments. We emphasize in addition, that in the practical PITM numerical simulation, one does solve equation (12) in its unsteady instantaneous form and not equation (13) which is written in a statistical form. However, equation (12) indeed contains all the information of equation (13). These two equations are not antithetical, the first one contains the second one. To insure consistency, the fluctuating advection terms in the unsteady equations can be considered as a non-zero mean value (physically interpreted as a diffusion term) plus a purely fluctuating part with zero mean. The only difference is that the diffusion term $\left\langle u_{j}^{<} k^{(s)}\right\rangle$ and the production term $F^{(1)}$ due to macro-fluctuations are simulated in the first case and are modeled in statistical approaches like multiscale models but they are present in both. 


\section{The control function in non-homogeneous turbulent flows}

In a general point of view, one can consider the control function $R$ as a function of $r=\left\langle k^{(s)}\right\rangle / k$ such that $0 \leqslant R(r) \leqslant 1$ appearing in Eq. (1) in the dissipation-rate transport equation represented as an adjustable varying parameter in the source term. Formulated in such a way [31, 32, 33], the issue to address is then to determine the variation in the solution of the turbulence model induced by a given variation in this control parameter.

\subsection{Turbulence transport equations in non-homogeneous flows}

We demonstrate in this section that the value $R=r$ found in locally homogeneous flows in the framework of the PITM method still remains valid in non-homogeneous turbulent flows. This result is not obvious and is not necessarily true for any subgrid scale model. Indeed, the work of Heinz et al. [31, 32, 33] based on a variational approach has shown that very different results can be obtained depending on the model considered. In some cases very strong variations can be obtained such as $R=(\Delta / L)^{2}=\eta_{c}^{-2}$ corresponding to $R=r^{3}$ which is very different from $R=r$, in particular for small values of $r$. This result seems to be dependent on the formulation of the transport equations of each particular model. We shall restrict ourselves here to the PITM model. Let us consider now, the PITM model in the simpler kinetic energy form with eddy viscosity coefficient. This choice is made for simplicity in the present purpose, but second order modeling would also lead to the same results. The modeled unsteady kinetic energy equation corresponding to the exact instantaneous equation (12) that is solved in the PITM simulation reads

$$
\frac{\mathcal{D} k^{(s)}}{\mathcal{D} t}=P^{(s)}-\varepsilon^{(s)}+J_{k}^{(s)}\left(k^{(s)}\right)
$$

where

$$
J_{k}^{(s)}\left(k^{(s)}\right)=C_{k} \frac{\partial}{\partial x_{j}}\left(\frac{k^{(s)^{2}}}{\varepsilon^{(s)}} \frac{\partial k^{(s)}}{\partial x_{j}}\right)
$$

The numerical simulation using this equation produces a fluctuating solution including the eddying large scales. It is then possible to operate a statistical averaging to this solution in order to get the mean values of the calculated quantities. Mathematically, these mean values also verify the mean equation developed below. Unlike in the modeled equation for the mean kinetic energy, here some terms are 
obtained from averaging of resolved quantities and not modeled. The averaging in a statistical sense of equation (29) is then

$$
\left\langle\frac{\mathcal{D} k^{(s)}}{\mathcal{D} t}\right\rangle=\left\langle P^{(s)}\right\rangle-\left\langle\varepsilon^{(s)}\right\rangle+\left\langle J_{k}^{(s)}\left(k^{(s)}\right)\right\rangle
$$

in which the diffusivity term using gradient approximation as been slightly approximated considering that

$$
\left\langle J_{k}^{(s)}\left(k^{(s)}\right)\right\rangle=C_{k}\left\langle\frac{k^{(s)^{2}}}{\varepsilon^{(s)}} \frac{\partial k^{(s)}}{\partial x_{j}}\right\rangle \approx C_{k} \frac{\left\langle k^{(s)}\right\rangle^{2}}{\left\langle\varepsilon^{(s)}\right\rangle} \frac{\partial\left\langle k^{(s)}\right\rangle}{\partial x_{j}}
$$

The companion RANS model equation would be

$$
\frac{D k}{D t}=P-\epsilon+C_{k} \frac{\partial}{\partial x_{j}}\left(\frac{k^{2}}{\epsilon} \frac{\partial k}{\partial x_{j}}\right)
$$

and

$$
\frac{D \epsilon}{D t}=C_{\epsilon_{1}} \frac{P}{\epsilon}-C_{\epsilon_{2}} \frac{\epsilon^{2}}{k}+C_{\epsilon} \frac{\partial}{\partial x_{j}}\left(\frac{k^{2}}{\epsilon} \frac{\partial \epsilon}{\partial x_{j}}\right)
$$

where $k$ and $\varepsilon$ are full statistical quantities. The production term appearing in equation (31) is then developed as follows

$$
\begin{aligned}
\left\langle P^{(s)}\right\rangle=-\left\langle\tau_{i j}^{(s)} \frac{\partial \bar{u}_{j}}{\partial x_{j}}\right\rangle & =-\left\langle\tau_{i j}^{(s)}\right\rangle \frac{\partial\left\langle u_{j}\right\rangle}{\partial x_{j}}-\left\langle\tau_{i j}^{(s)} \frac{\partial u_{j}^{<}}{\partial x_{j}}\right\rangle \\
& \approx 2 \nu_{t}^{(s)}\left\langle S_{i j}\right\rangle\left\langle S_{i j}\right\rangle-\left\langle\tau_{i j}^{(s)} \frac{\partial u_{j}^{<}}{\partial x_{j}}\right\rangle
\end{aligned}
$$

with

$$
\left\langle\tau_{i j}^{(s)} \frac{\partial u_{j}^{<}}{\partial x_{j}}\right\rangle=\left\langle\tau_{i j}^{(s)} \frac{\partial u_{j}^{<}}{\partial x_{j}}\right\rangle+\left\langle\tau_{i j}^{(s)} \frac{\partial u_{j}^{<}}{\partial x_{j}}\right\rangle \approx\left\langle\tau_{i j}^{(s)} \frac{\partial u_{j}^{<}}{\partial x_{j}}\right\rangle
$$

because as recalled above, the double correlation between quantities pertaining to disjoint spectral zones vanishes when using a spectral cutoff filter [22]. Secondly, combining Eq. (27) with Eq. (31) leads to

$$
\frac{D\left\langle k^{(s)}\right\rangle}{D t}=\left\langle P^{(s)}\right\rangle-\left\langle\varepsilon^{(s)}\right\rangle+C_{k} \frac{\partial}{\partial x_{j}}\left(\frac{\left\langle k^{(s)}\right\rangle^{2}}{\left\langle\varepsilon^{(s)}\right\rangle} \frac{\partial\left\langle k^{(s)}\right\rangle}{\partial x_{j}}\right)-\frac{\partial}{\partial x_{j}}\left\langle u_{j}^{<} k^{(s)}\right\rangle
$$

The diffusion process by the large scale fluctuating velocity $u_{j}^{<}$can be readily approximated from the usual gradient hypothesis (see Appendix D)

$$
\left\langle J_{k}^{(r)}\left(k^{(s)}\right)\right\rangle=-\frac{\partial}{\partial x_{j}}\left(\left\langle u_{j}^{<} k^{(s)}\right)\right\rangle \approx C_{k} \frac{\partial}{\partial x_{j}}\left(\frac{\left\langle k^{(r)}\right\rangle^{2}}{\left\langle\varepsilon^{(s)}\right\rangle} \frac{\partial\left\langle k^{(s)}\right\rangle}{\partial x_{j}}\right)
$$


so that last term appearing in (37) can be computed as

$$
\frac{\partial}{\partial x_{j}}\left[\left\langle u_{j}^{<} k^{(s)}\right\rangle\right] \approx-C_{k} \frac{\partial}{\partial x_{j}}\left(\frac{\left\langle k^{(r)}\right\rangle^{2}}{\left\langle\varepsilon^{(s)}\right\rangle} \frac{\partial\left\langle k^{(s)}\right\rangle}{\partial x_{j}}\right)
$$

where $k^{(r)}$ denote the resolved turbulence kinetic energy defined as $k^{(r)}=\left(\bar{u}_{i} \bar{u}_{i}-\left\langle u_{i}\right\rangle\left\langle u_{i}\right\rangle\right) / 2$ as defined in Ref. [22]. As a result of modeling considering also that $\left\langle\varepsilon^{(s)}\right\rangle \approx \epsilon$, the total statistical diffusion term appears

$$
C_{k} \frac{\partial}{\partial x_{j}}\left(\frac{\left\langle k^{(s)}\right\rangle^{2}}{\left\langle\epsilon^{(s)}\right\rangle} \frac{\partial\left\langle k^{(s)}\right\rangle}{\partial x_{j}}\right)-\frac{\partial}{\partial x_{j}}\left[\left\langle k^{(s)} u_{j}^{<}\right\rangle\right] \approx C_{k} \frac{\partial}{\partial x_{j}}\left[\frac{k^{2}}{\epsilon} \frac{\partial\left\langle k^{(s)}\right\rangle}{\partial x_{j}}\right]
$$

where it is recalled that $k^{(s+r)}=k^{(s)}+k^{(r)}$ and $k=\left\langle k^{(s+r)}\right\rangle=\left\langle k^{(s)}\right\rangle+\left\langle k^{(r)}\right\rangle$ (see Appendix A) leading to the resulting equation

$$
\frac{D\left\langle k^{(s)}\right\rangle}{D t}=\left\langle P^{(s)}\right\rangle-\left\langle\varepsilon^{(s)}\right\rangle+\left\langle J_{k}^{(s+r)}\left(k^{(s)}\right)\right\rangle
$$

where we have denoted

$$
\left\langle J_{k}^{(s+r)}\left(k^{(s)}\right)\right\rangle=\left\langle J_{k}^{(s)}\left(k^{(s)}\right)\right\rangle+\left\langle J_{k}^{(r)}\left(k^{(s)}\right)\right\rangle
$$

giving

$$
\left\langle J_{k}^{(s+r)}\left(k^{(s)}\right)\right\rangle \approx C_{k} \frac{\partial}{\partial x_{j}}\left(\frac{k^{2}}{\varepsilon} \frac{\partial\left\langle k^{(s)}\right\rangle}{\partial x_{j}}\right)
$$

Considering now the instantaneous unsteady dissipation-rate equation that is solved in the PITM framework, the same reasoning as previously can be made

$$
\frac{\mathcal{D} \varepsilon^{(s)}}{\mathcal{D} t}=C_{\epsilon_{1}}^{(s)} \frac{P^{(s)} \varepsilon^{(s)}}{k^{(s)}}-C_{\epsilon_{2}}^{(s)} \frac{\varepsilon^{(s)^{2}}}{k^{(s)}}+J_{\epsilon}^{(s)}\left(\epsilon^{(s)}\right)
$$

where

$$
J_{\epsilon}^{(s)}\left(\epsilon^{(s)}\right)=C_{\epsilon} \frac{\partial}{\partial x_{j}}\left(\frac{k^{(s)^{2}}}{\varepsilon^{(s)}} \frac{\partial \epsilon^{(s)}}{\partial x_{j}}\right)
$$

and $C_{\epsilon_{1}}^{(s)}$ and $C_{\epsilon_{2}}^{(s)}$ are given by Eqs.(6) and (5), respectively. Like in equation (29), we now operate statistical averaging on the numerical simulation results in order to get the mean value. This mean value satisfies also the mean equation developed below in which some terms are obtained from averaging of resolved quantities and not modeled. Thus, the averaging of Eq. (44) using some very acceptable approximations leads to

$$
\left\langle\frac{\mathcal{D} \varepsilon^{(s)}}{\mathcal{D} t}\right\rangle=C_{\epsilon_{1}}^{(s)} \frac{\left\langle P^{(s)}\right\rangle\left\langle\varepsilon^{(s)}\right\rangle}{\left\langle k^{(s)}\right\rangle}-C_{\epsilon_{2}}^{(s)} \frac{\left\langle\varepsilon^{(s)}\right\rangle^{2}}{\left\langle k^{(s)}\right\rangle}+\left\langle J_{\epsilon}^{(s)}\left(\epsilon^{(s)}\right)\right\rangle
$$


where

$$
\left\langle J_{\epsilon}^{(s)}\left(\epsilon^{(s)}\right)\right\rangle=C_{\epsilon}\left\langle\frac{\partial}{\partial x_{j}}\left(\frac{k^{(s)^{2}}}{\varepsilon^{(s)}} \frac{\partial \epsilon^{(s)}}{\partial x_{j}}\right)\right\rangle \approx C_{\varepsilon} \frac{\partial}{\partial x_{j}}\left(\frac{\left\langle k^{(s)}\right\rangle^{2}}{\left\langle\varepsilon^{(s)}\right\rangle} \frac{\partial\left\langle\varepsilon^{(s)}\right\rangle}{\partial x_{j}}\right)
$$

Applying (152) with $\phi=\varepsilon^{(s)}$, one can obtain

$$
\frac{D\left\langle\varepsilon^{(s)}\right\rangle}{D t}=\left\langle\frac{\mathcal{D} \varepsilon^{(s)}}{\mathcal{D} t}\right\rangle-\frac{\partial}{\partial x_{j}}\left\langle u_{j}^{<} \varepsilon^{(s)}\right\rangle
$$

so that

$$
\begin{aligned}
& \frac{D\left\langle\varepsilon^{(s)}\right\rangle}{D t}=C_{\epsilon_{1}}^{(s)} \frac{\left\langle P^{(s)}\right\rangle\left\langle\varepsilon^{(s)}\right\rangle}{\left\langle k^{(s)}\right\rangle}-C_{\epsilon_{2}}^{(s)} \frac{\left\langle\varepsilon^{(s)}\right\rangle^{2}}{\left\langle k^{(s)}\right\rangle}+C_{\varepsilon} \frac{\partial}{\partial x_{j}}\left(\frac{\left\langle k^{(s)}\right\rangle^{2}}{\left\langle\varepsilon^{(s)}\right\rangle} \frac{\partial\left\langle\varepsilon^{(s)}\right\rangle}{\partial x_{j}}\right) \\
& -\frac{\partial}{\partial x_{j}}\left\langle u_{j}^{<} \varepsilon^{(s)}\right\rangle
\end{aligned}
$$

The total diffusion is involving all the length scales, as explained in section 1 . This fact was earlier recognized by Schiestel [38] (see section \$19.14.3). To move forward in the reasoning, we need an approximation of the total diffusion. Using for instance a diffusivity hypothesis like in usual statistical closures, one can suppose realistically (see Appendix D)

$$
\left\langle J_{\epsilon}^{(r)}\left(\epsilon^{(s)}\right)\right\rangle=-\frac{\partial}{\partial x_{j}}\left\langle u_{j}^{<} \epsilon^{(s)}\right\rangle \approx C_{\epsilon} \frac{\partial}{\partial x_{j}}\left(\frac{\left\langle k^{(r)}\right\rangle^{2}}{\left\langle\varepsilon^{(s)}\right\rangle} \frac{\partial\left\langle\epsilon^{(s)}\right\rangle}{\partial x_{j}}\right)
$$

Of course, this approximation is not intended to be used in numerical PITM simulation, but only to get a useful estimate in the present purpose. Finally, one can infer approximately

$$
C_{\varepsilon} \frac{\partial}{\partial x_{j}}\left(\frac{\left\langle k^{(s)}\right\rangle^{2}}{\left\langle\varepsilon^{(s)}\right\rangle} \frac{\partial\left\langle\varepsilon^{(s)}\right\rangle}{\partial x_{j}}\right)-\frac{\partial}{\partial x_{j}}\left\langle u_{j}^{<} \varepsilon^{(s)}\right\rangle \approx C_{\varepsilon} \frac{\partial}{\partial x_{j}}\left(\frac{k^{2}}{\varepsilon} \frac{\partial\left\langle\varepsilon^{(s)}\right\rangle}{\partial x_{j}}\right)
$$

just confirming that all the scales contribute to the turbulent diffusion of the mean value, including both the modeled small scales and the resolved scales. Combining Eq. (49) with Eq. (51) yields the transport equation for $\left\langle\varepsilon^{(s)}\right\rangle$ as

$$
\frac{D\left\langle\epsilon^{(s)}\right\rangle}{D t}=C_{\epsilon_{1}}^{(s)} \frac{\left\langle P^{(s)}\right\rangle\left\langle\varepsilon^{(s)}\right\rangle}{\left\langle k^{(s)}\right\rangle}-C_{\epsilon_{2}}^{(s)} \frac{\left\langle\varepsilon^{(s)}\right\rangle^{2}}{\left\langle k^{(s)}\right\rangle}+\left\langle J_{\epsilon}^{(s+r)}\left(\epsilon^{(s)}\right)\right\rangle
$$

where we have denoted

$$
\left\langle J_{\epsilon}^{(s+r)}\left(\epsilon^{(s)}\right)\right\rangle=\left\langle J_{\epsilon}^{(s)}\left(\epsilon^{(s)}\right)\right\rangle+\left\langle J_{\epsilon}^{(r)}\left(\epsilon^{(s)}\right)\right\rangle
$$


giving

$$
\left\langle J_{\epsilon}^{(s+r)}\left(\epsilon^{(s)}\right)\right\rangle \approx C_{\varepsilon} \frac{\partial}{\partial x_{j}}\left(\frac{k^{2}}{\epsilon} \frac{\partial\left\langle\varepsilon^{(s)}\right\rangle}{\partial x_{j}}\right)
$$

Thus, the usual equation of the statistical model in inhomogeneous flows is entirely recovered

$$
\frac{D \varepsilon}{D t}=C_{\epsilon_{1}}^{(s)} \frac{\left\langle P^{(s)}\right\rangle \epsilon}{\left\langle k^{(s)}\right\rangle}-C_{\epsilon_{2}}^{(s)} \frac{\varepsilon^{2}}{\left\langle k^{(s)}\right\rangle}+C_{\varepsilon} \frac{\partial}{\partial x_{j}}\left(\frac{k^{2}}{\varepsilon} \frac{\partial \varepsilon}{\partial x_{j}}\right)
$$

with as usual

$$
C_{\epsilon_{2}}^{(s)}=C_{\epsilon_{1}}^{(s)}+\frac{\left\langle k^{(s)}\right\rangle}{k}\left(C_{\epsilon_{2}}-C_{\epsilon_{1}}\right)
$$

and

$$
C_{\epsilon_{1}}^{(s)}=C_{\epsilon_{1}}
$$

It is of interest to note that the diffusion term appearing in Eq. (55) is in fact, and in spite of appearances, no longer dependent on the cutoff wave number $\kappa_{c}$. Incidentally, one can remark that in statistical multiple scales models the transport equations of partial energies do make use of the total turbulent diffusivity as evidenced here in Eq. (55). Practical formulations of the statistical multiple scale models also keep this feature [38] or partial Reynolds stress models giving the diffusivity

$$
\sigma^{(m)}=c_{s}^{(m)} \frac{k R_{i j}}{F^{(m)}}
$$

in the partial $(m)$ zone and for partial kinetic energy models giving the diffusivity

$$
\sigma^{(m)}=c_{s}^{(m)} \frac{k^{2}}{F^{(m)}}
$$

in the partial $(m)$ zone. In these formulas $k$ stands for the total statistical kinetic energy of turbulence.

\subsection{The equilibrium logarithmic layer}

For a clear physical appraisal we consider particularizing the previous equations to the equilibrium logarithmic layer. As pointed out by Hamba [39], hybrid RANS/LES models often mismatch the logaritmic layer that is nevertheless crucial to reproduce accurately for internal flows. The particular case of the logarithmic turbulent boundary layer is easy to handle analytically and is then suitable to show the expected behavior of a typical non-homogeneous flow with direct physical interpretation. We 
shall extend the reasoning to general inhomogeneous flow afterwards. We consider here steady flows in the boundary layer close to equilibrium state verifying $\left\langle P^{(s)}\right\rangle \approx P$ as well as $\left\langle\varepsilon^{(s)}\right\rangle \approx \epsilon$ as explained in Appendix C. The mean velocity profile is given by the logarithmic law of the wall as

$$
\left\langle u_{1}\right\rangle=\frac{u_{\tau}}{K} \operatorname{Ln}\left(E x_{n}\right)
$$

where $x_{n}$ denotes the normal coordinate to the wall, $u_{\tau}$ is the friction velocity, $K$ the Kármán constant. The derivative of Eq. (60) is then

$$
\frac{\partial\left\langle u_{1}\right\rangle}{\partial x_{n}}=\frac{u_{\tau}}{K x_{n}}
$$

The turbulence field is in energetic equilibrium, as a consequence of the mean kinetic energy equation, so that

$$
\varepsilon=P=u_{\tau}^{2} \frac{\partial\left\langle u_{1}\right\rangle}{\partial x_{n}}=\frac{u_{\tau}^{3}}{K x_{n}}
$$

The kinetic energy $k$ in a logarithmic layer is not dependent upon $x_{n}$ and more precisely its value can be classically related to the friction velocity by the relation

$$
k=\frac{u_{\tau}^{2}}{\sqrt{C_{\mu}}}
$$

In RANS modeling, Eq. (33) and (34) lead to the resulting equation

$$
\left(C_{\epsilon_{1}}-C_{\epsilon_{2}}\right) \frac{\epsilon^{2}}{k}+C_{\epsilon} \frac{\partial}{\partial x_{n}}\left(\frac{k^{2}}{\epsilon} \frac{\partial \epsilon}{\partial x_{n}}\right)=0 .
$$

On the other hand, Eq. (55) yields

$$
\left(C_{\epsilon_{1}}^{(s)}-C_{\epsilon_{2}}^{(s)}\right) \frac{\epsilon^{2}}{\left\langle k^{(s)}\right\rangle}+C_{\epsilon} \frac{\partial}{\partial x_{n}}\left(\frac{k^{2}}{\epsilon} \frac{\partial \epsilon}{\partial x_{n}}\right)=0 .
$$

Comparing Eq. (64) with Eq. (65) allows to recover equation (56) showing that the control function $R$ introduced in the more general case in Eq. (1) verifies $R=r$. So, this solution associated with the PITM method is still valid in the logarithmic layer for the PITM method.

We now consider the system of equations (29) and (44) describing the evolution of the unsteady quantities for the PITM model $k^{(s)}$ and $\epsilon^{(s)}$ in the turbulent flow. In this case, neglecting the fluctuations around the mean values, and assuming (erroneously) local equilibrium along streamlines of the filtered field would falsely lead to the resulting equation

$$
\left(C_{\epsilon_{1}}^{(s)}-C_{\epsilon_{2}}^{(s)}\right) \frac{\epsilon^{2}}{k^{(s)}}+C_{\epsilon} \frac{\partial}{\partial x_{n}}\left(\frac{r^{2} k^{2}}{\epsilon} \frac{\partial \epsilon}{\partial x_{n}}\right)=0 .
$$


Considering that $r$ is constant in the logarithmic zone, Eq. (66) associated with Eqs. (1) and (64) would yield the result $R=r^{3}$ found by Heinz et al. [31,32]. But this argument is not relevant in the case of the PITM method because the instantaneous flow is unsteady and local equilibrium is acceptable only in the statistical sense. In this case, Eq. (66) in the mean value would not be consistent with the corresponding statistical equation. This is explained further in the next section.

\subsection{Physical interpretation}

This result concerning the different values for $R$ can be interpreted from a physical point of view. The equations solved in a practical PITM numerical simulation are indeed equations (29) and (44) which give instantaneous fluctuating quantities including large scale fluctuations. As explained in the previous sections, one is free to make statistical averaging of the solution obtained from the simulation. These mean values mathematically satisfy the mean equations (31) and (46). So, one can say that both equations (unaveraged ones and averaged ones) are satisfied. The unaveraged equations (29) and (44) can be understood as composed of the averaged equations (31) and (46) plus their fluctuating counterpart. From Eq. (172) of Appendix D, this reads

$$
-\frac{\partial}{\partial x_{j}}\left(u_{j}^{<} \psi^{<}\right)=\left\langle J_{\psi}^{(r)}\right\rangle+J_{\psi}^{(r)}<C_{\psi} \frac{\partial}{\partial x_{j}}\left[\frac{\left\langle k^{(r)}\right\rangle^{2}}{\left\langle\epsilon^{(s)}\right\rangle} \frac{\partial\langle\psi\rangle}{\partial x_{j}}\right]+J_{\psi}^{(r)}<
$$

This term cannot be zero, even in steady flows. We have thus shown that equations (29) and (44) both include a resolved diffusion term coming from the advection term in the instantaneous field but corresponding to the diffusion by large eddies in the averaged field. As also indicated in Appendix D, this term can be estimated so that the apparent diffusion coefficient is no longer $k^{(s) 2} / \varepsilon$, like one can suppose erroneously from Eq. (44) but indeed $k^{2} / \varepsilon$ from a physical standpoint. This is because the inertial terms in the instantaneous unsteady equations have a non zero mean value corresponding to an additional term in the diffusion process which is present both in the unsteady and mean equations. This is the key of the demonstration that yields $R(r)=r$ in all cases. It is a physical property of the turbulent solution that cannot be discarded. The application to the equilibrium logarithmic layer simply emphasizes the importance of these terms. 


\section{$4 \quad$ Variational analysis}

\subsection{General derivation}

The objective of this section is to provide more general elements of proof leading to $R(r)=r$ as already obtained in the preceding section. For that purpose, we conduct at variational analysis of the turbulence equations. This approach was used previously by Heinz et al. [31, 32, 33] in their developments giving rise to the result $R(r)=r^{3}$ which is in contradiction with the present result $R(r)=r$. The main reason arises from the model equations and the unsteady behavior of their solutions. Heinz et al. [31, 32, 33] have considered the instantaneous equations (29) and (44) describing the evolution of the unsteady quantities in the PITM model $k^{(s)}$ and $\epsilon^{(s)}$ in the flow with a statistical interpretation that would be valid only for the mean equations (41) and (52). The idea behind the reasoning in the use of variational calculus is to find an analytical link between a small variation in the model coefficient $C_{\epsilon_{2}}^{(s)}$ (the method could be conceived to apply to other coefficients as well) and the correlative changes induced in the solution. Introducing a small numerical variation $\delta C_{\epsilon_{2}}^{(s)}$ on the $C_{\epsilon_{2}}^{(s)}$ coefficient, the simulation results will change accordingly. The changes in the solution occur in a functional space and thus are represented as functional variations in the turbulent field. So, we define the corresponding virtual functional variations as $\delta k^{(s)}=\zeta_{k}\left(x_{i}, t\right) \delta a$ and $\delta \epsilon^{(s)}=\zeta_{\varepsilon}\left(x_{i}, t\right) \delta b$ for energy and dissipation, where $\zeta_{k}\left(x_{i}, t\right)$ and $\zeta_{\epsilon}\left(x_{i}, t\right)$ are arbitrary functions in space and time (with appropriate boundary conditions) and $\delta a, \delta b$, small scalar increments, the resulting link between these variations is obtained from functional differentiation of the corresponding transport equations. The reasoning has to be conducted primarily on the instantaneous equations which are effectively solved in the simulation. But, one has to account for the statistical properties of the solution which are also compatible with the statistical equations (which are not solved directly but have to be verified has a consequence). So, in the energy equation (29) equilibrium is assumed along mean streamlines (and not along instantaneous streamlines). So, this hypothesis implies that the turbulent field is near equilibrium in a statistical sense. In the following, like in Heinz [32], we shall work on the instantaneous equations describing the large scale fluctuating turbulent resolved simulation but with the constant concern that these equations have to be consistent with their averaged counterpart. We emphasize again that we have not to choose between the two types of equations (instantaneous or averaged) because they have to produce consistent results. Using the formal operator 
defined in Eq. (148), Eq. (29) can be rewritten as

$$
\frac{D k^{(s)}}{D t}=P^{(s)}-\epsilon^{(s)}+J_{k}^{(s)}\left(k^{(s)}\right)-\frac{\partial}{\partial x_{j}}\left(u_{j}^{<} k^{(s)}\right)
$$

where as given by Eq. (145),

$$
\frac{D k^{(s)}}{D t}=\frac{\partial k^{(s)}}{\partial t}+\left\langle u_{j}\right\rangle \frac{\partial k^{(s)}}{\partial x_{j}}
$$

is the derivative along mean streamlines. Remark that $u_{j}^{<} k^{(s)}$ could be written also $\overline{u_{j}^{<} k^{(s)}}$ only in the special case of a top hat filter in physical space. The last term of the RHS in the latter Eq. (68) will be simulated from the resolved scales calculation but it is possible to get a straightforward approximation of its magnitude by using the gradient diffusion hypothesis introduced in Eq. (38). Then, this equation in the mean reads in a more compact form as

$$
\frac{D\left\langle k^{(s)}\right\rangle}{D t}=\left\langle P^{(s)}\right\rangle-\left\langle\epsilon^{(s)}\right\rangle+\left\langle J_{k}^{(s+r)}\left(k^{(s)}\right)\right\rangle
$$

where according to Eq. (42), $\left\langle J_{k}^{(s+r)}\left(k^{(s)}\right)\right\rangle=\left(\left\langle J_{k}^{(s)}\right\rangle+\left\langle J_{k}^{(r)}\right\rangle\right)\left(k^{(s)}\right)$. The mean value of the LHS of Eq. (29) is not zero, because the advection term once averaged gives a part of the diffusion as detailed in appendix D. If the LHS of Eq. (29) was taken equal to zero, the averaging of equation (68) or (70) would not be exact and consistent with the well known physical phenomenology of fluid turbulence. It would be nonphysical to discard this term. Similarly, using again the formal operator defined in Eq. (148) introducing the derivative along mean streamlines, Eq. (44) can be rewritten as

$$
\frac{D \varepsilon^{(s)}}{D t}=C_{\epsilon_{1}}^{(s)} \frac{P^{(s)} \varepsilon^{(s)}}{k^{(s)}}-C_{\epsilon_{2}}^{(s)} \frac{\varepsilon^{(s)^{2}}}{k^{(s)}}+J_{\epsilon}^{(s)}\left(\epsilon^{(s)}\right)-\frac{\partial}{\partial x_{j}}\left(u_{j}^{<} \epsilon^{(s)}\right)
$$

Like previously, the LHS of equation (44) has a non zero mean value, it gives rise to a non zero diffusion term. Using also the gradient diffusion approximation introduced in Eq. (50), Eq. (71) then reads in a compact form as

$$
\frac{D\left\langle\varepsilon^{(s)}\right\rangle}{D t}=C_{\epsilon_{1}}^{(s)} \frac{\left\langle P^{(s)}\right\rangle\left\langle\varepsilon^{(s)}\right\rangle}{\left\langle k^{(s)}\right\rangle}-C_{\epsilon_{2}}^{(s)} \frac{\left\langle\varepsilon^{(s)}\right\rangle^{2}}{k^{(s)}}+\left\langle J_{\epsilon}^{(s+r)}\left(\epsilon^{(s)}\right)\right\rangle
$$

where according to Eq. (53), $\left\langle J_{\epsilon}^{(s+r)}\left(\epsilon^{(s)}\right)\right\rangle=\left(\left\langle J_{\epsilon}^{(s)}\right\rangle+\left\langle J_{\epsilon}^{(r)}\right\rangle\right)\left(\epsilon^{(s)}\right)$. At this step, it is useful to recall the general expression of the diffusion term given by Eq. (177) as

$$
\left\langle J_{\psi}^{(s+r)}(\psi)\right\rangle \approx C_{\psi} \frac{\partial}{\partial x_{j}}\left[\left\langle\frac{\left(k^{(s)}+k^{(r)}\right)^{2}}{\epsilon^{(s)}}\right\rangle \frac{\partial\langle\psi\rangle}{\partial x_{j}}\right] \approx C_{\psi} \frac{\partial}{\partial x_{j}}\left[\frac{\left(\left\langle k^{(s+r)}\right\rangle\right)^{2}}{\left\langle\epsilon^{(s)}\right\rangle} \frac{\partial\langle\psi\rangle}{\partial x_{j}}\right]
$$


where $\psi$ denotes either $k^{(s)}$ or $\epsilon^{(s)}$. Considering that the turbulent field remains near equilibrium along mean streamlines, $D\left\langle k^{(s)}\right\rangle / D t=D\left\langle\epsilon^{(s)}\right\rangle / D t=0$ leads to the two balance equations

$$
\left\langle P^{(s)}\right\rangle=\left\langle\epsilon^{(s)}\right\rangle-\left\langle J_{k}^{(s+r)}\left(k^{(s)}\right)\right\rangle
$$

and

$$
C_{\epsilon_{1}}^{(s)}\left\langle P^{(s)}\right\rangle-C_{\epsilon_{2}}^{(s)}\left\langle\epsilon^{(s)}\right\rangle=-\frac{\left\langle k^{(s)}\right\rangle}{\left\langle\epsilon^{(s)}\right\rangle}\left\langle J_{\epsilon}^{(s+r)}\left(\epsilon^{(s)}\right)\right\rangle
$$

Remark that the hypothesis of near equilibrium along mean streamlines would not be physically correct if applied to instantaneous values. Indeed, for instance in the equilibrium turbulent logarithmic layer derivatives of turbulent quantities along mean stream lines vanish while it is not true at all for filtered quantities which always fluctuate. Combining Eq. (74) with Eq. (75) by eliminating $\left\langle P^{(s)}\right\rangle$ yields the resulting equation

$$
C_{\epsilon_{2}}^{(s)}-C_{\epsilon_{1}}^{(s)}=\frac{\left\langle k^{(s)}\right\rangle}{\left\langle\epsilon^{(s)}\right\rangle^{2}}\left\langle J_{\epsilon}^{(s+r)}\left(\epsilon^{(s)}\right)\right\rangle\left[1-C_{\epsilon_{1}}^{(s)} \frac{\left\langle\epsilon^{(s)}\right\rangle}{\left\langle k^{(s)}\right\rangle} \frac{\left\langle J_{k}^{(s+r)}\left(k^{(s)}\right)\right\rangle}{\left\langle J_{\epsilon}^{(s+r)}\left(\epsilon^{(s)}\right)\right\rangle}\right]
$$

Now, taking the functional variation gives

$$
\frac{\delta C_{\epsilon_{2}}^{(s)}}{C_{\epsilon_{2}}^{(s)}-C_{\epsilon_{1}}^{(s)}}=\frac{\delta\left\langle k^{(s)}\right\rangle}{\left\langle k^{(s)}\right\rangle}-2 \frac{\delta\left\langle\epsilon^{(s)}\right\rangle}{\left\langle\epsilon^{(s)}\right\rangle}+\frac{\delta\left\langle J_{\epsilon}^{(s+r)}\left(\epsilon^{(s)}\right)\right\rangle}{\left\langle J_{\epsilon}^{(s+r)}\left(\epsilon^{(s)}\right)\right\rangle}+\frac{\delta E}{E}
$$

where the function $E$ is given by

$$
E=1-C_{\epsilon_{1}}^{(s)} \frac{\left\langle\epsilon^{(s)}\right\rangle}{\left\langle k^{(s)}\right\rangle} \frac{\left\langle J_{k}^{(s+r)}\left(k^{(s)}\right)\right\rangle}{\left\langle J_{\epsilon}^{(s+r)}\left(\epsilon^{(s)}\right)\right\rangle}
$$

At this stage, it is necessary to calculate the variation of the diffusion terms $\delta J_{k}^{(s+r)}\left(k^{(s)}\right)$ and $\delta J_{\epsilon}^{(s+r)}\left(\epsilon^{(s)}\right)$ as well as $\delta E$. From Eq. (73), one can obtain in a first order approximation (see appendix E) the result

$$
\frac{\delta\left\langle J_{\psi}^{(s+r)}(\psi)\right\rangle}{\left\langle J_{\psi}^{(s+r)}(\psi)\right\rangle} \approx 2 \frac{\delta\left\langle k^{(s+r)}\right\rangle}{\left\langle k^{(s+r)}\right\rangle}-\frac{\delta\left\langle\epsilon^{(s)}\right\rangle}{\left\langle\epsilon^{(s)}\right\rangle}+\frac{\delta\langle\psi\rangle}{\langle\psi\rangle} \approx-\frac{\delta\left\langle\epsilon^{(s)}\right\rangle}{\left\langle\epsilon^{(s)}\right\rangle}+\frac{\delta\langle\psi\rangle}{\langle\psi\rangle}
$$

because $\delta\left\langle k^{(s+r)}\right\rangle=\delta\left\langle k^{(s)}\right\rangle+\delta\left\langle k^{(r)}\right\rangle \approx 0$ as the averaged total turbulence energy $k=\left\langle k^{(s+r)}\right\rangle$ remains almost constant and independent of the grid, characterized in the spectral space by the spectral cutoff 
location $\kappa_{c}$. Indeed, in the case where the grid step size increases, then a part of the energy contained into the resolved scales is removed and fed into the modeled spectral zone, whereas on the contrary, when the grid step size decreases, a part of energy coming from the modeled zone is injected into the resolved scales $[35,36]$. This is the hypothesis of self-consistency of the model which has been verified in practical simulations, this hypothesis is also currently made by other authors [31, 32, 33]. Thus, the diffusion coefficient acting in these equations involves the total kinetic energy and not the partial energy. For $\psi=k^{(s)}$ and $\psi=\epsilon^{(s)}$, respectively, Eq. (79) implies

$$
\frac{\delta\left\langle J_{k}^{(s+r)}\left(k^{(s)}\right)\right\rangle}{\left\langle J_{k}^{(s+r)}\left(k^{(s)}\right)\right\rangle} \approx \frac{\delta\left\langle k^{(s)}\right\rangle}{\left\langle k^{(s)}\right\rangle}-\frac{\delta\left\langle\epsilon^{(s)}\right\rangle}{\left\langle\epsilon^{(s)}\right\rangle} \text { and } \frac{\delta\left\langle J_{\epsilon}^{(s+r)}\left(\epsilon^{(s)}\right)\right\rangle}{\left\langle J_{\epsilon}^{(s+r)}\left(\epsilon^{(s)}\right)\right\rangle} \approx 0
$$

The variation of the function $E$ reads

$$
\frac{\delta E}{E-1}=\frac{\delta\left\langle\epsilon^{(s)}\right\rangle}{\left\langle\epsilon^{(s)}\right\rangle}-\frac{\delta\left\langle k^{(s)}\right\rangle}{\left\langle k^{(s)}\right\rangle}+\frac{\delta\left\langle J_{k}^{(s+r)}\left(k^{(s)}\right)\right\rangle}{\left\langle J_{k}^{(s+r)}\left(k^{(s)}\right)\right\rangle}-\frac{\delta\left\langle J_{\epsilon}^{(s+r)}\left(\epsilon^{(s)}\right)\right\rangle}{\left\langle J_{\epsilon}^{(s+r)}\left(\epsilon^{(s)}\right)\right\rangle}
$$

By substituting the expressions of $\delta\left\langle J_{k}^{(s+r)}\left(k^{(s)}\right)\right\rangle$ and $\delta\left\langle J_{\epsilon}^{(s+r)}\left(\epsilon^{(s)}\right)\right\rangle$ given by Eq. (80) into Eq.(81), it is found that

$$
\delta E \approx 0
$$

As a main result, Eq. (77) reduces finally to the simple expression

$$
\frac{\delta C_{\epsilon 2}^{(s)}}{C_{\epsilon 2}^{(s)}-C_{\epsilon 1}^{(s)}}=\frac{\delta\left\langle k^{(s)}\right\rangle}{\left\langle k^{(s)}\right\rangle}-2 \frac{\delta\left\langle\epsilon^{(s)}\right\rangle}{\left\langle\epsilon^{(s)}\right\rangle}
$$

This result is physically meaningful and was expected. Moreover, it can be verified from numerical simulations that varying $C_{\epsilon 2}^{(s)}$ modifies the subfilter turbulent energy level without modifying noticeably the total mean energy. As already mentioned, the increase (resp. decrease) of subfilter energy induces a decrease (resp. increase) of resolved energy such that the total amount remains unchanged. Considering that $\left\langle\epsilon^{(s)}\right\rangle$ remains not affected by the cutoff wave number, one obtains $\delta \varepsilon=0$. This sound practice has been made for instance in [40] and reflects the fact that the model is self-consistent that is to say that $\epsilon$ and $k$ are not appreciably influenced by the cutoff location, a fact that is verified in practical simulations. So that Eq. (83) leads to

$$
\frac{\delta C_{\epsilon 2}^{(s)}}{C_{\epsilon 2}^{(s)}-C_{\epsilon 1}^{(s)}}=\frac{\delta\left\langle k^{(s)}\right\rangle}{\left\langle k^{(s)}\right\rangle}
$$


which integrates as

$$
\int_{C_{\epsilon_{2}}^{(s)}}^{C_{\epsilon_{2}}} \frac{\delta C}{C-C_{\epsilon_{1}}^{(s)}}=\int_{\left\langle k^{(s)}\right\rangle}^{k} \frac{\delta\left\langle k^{(s)}\right\rangle}{\left\langle k^{(s)}\right\rangle}
$$

or as previously

$$
C_{\epsilon_{2}}^{(s)}-C_{\epsilon_{1}}^{(s)}=\frac{\left\langle k^{(s)}\right\rangle}{k}\left(C_{\epsilon_{2}}-C_{\epsilon_{1}}\right)
$$

that allows to recover Eq. (56) if $C_{\epsilon_{1}}^{(s)}=C_{\epsilon_{1}}$. This variational study has shown that the $C_{\epsilon_{2}}^{(s)}$ coefficient in the dissipation-rate equation is a tweaking parameter that allows to control the $\left\langle k^{(s)}\right\rangle / k$ ratio. Moreover this action is linear in the mathematical sense. It is important to note also that this result supposes that the subfilter model is consistent, that is to say that changing $C_{\epsilon_{2}}^{(s)}$ will indeed change the $\left\langle k^{(s)}\right\rangle / k$ ratio but the total mean turbulence energy (resolved + modeled) remains constant, this requirement is also assumed in other papers like in Refs. [31, 32, 33]. At this step, it is thus remarkable that the variational study, allows to recover the correct dependence of the $\epsilon$ equation coefficients in relation with the $k^{(s)} / k$ ratio as already obtained in the original PITM method in all cases, both homogeneous and non-homogeneous turbulence. Mathematically, this outcome put in light some functional aspects of the method in perfect agreement with the PITM derivation. It is worth noticing that the variational analysis reveals the intrinsic properties of the partial differential equations of the turbulence model and don't make any use of Eq. (8). The result given by Eq. (86) is thus totally independent of Eq. (8).

\subsection{What about the instantaneous quantities ?}

The instantaneous equations (68) and (71) accounting for the filtered variables can be rewritten as

$$
\frac{D\left\langle k^{(s)}\right\rangle}{D t}+\frac{D k^{(s)<}}{D t}=P^{(s)}-\epsilon^{(s)}+J_{k}^{(s)}\left(k^{(s)}\right)-\frac{\partial}{\partial x_{j}}\left(u_{j}^{<} k^{(s)}\right)
$$

and

$$
\frac{D\left\langle\epsilon^{(s)}\right\rangle}{D t}+\frac{D \epsilon^{(s)<}}{D t}=C_{\epsilon_{1}}^{(s)} \frac{P^{(s)} \epsilon^{(s)}}{k^{(s)}}-C_{\epsilon_{2}}^{(s)} \frac{\epsilon^{(s)^{2}}}{k^{(s)}}+J_{\epsilon}^{(s)}\left(\epsilon^{(s)}\right)-\frac{\partial}{\partial x_{j}}\left(u_{j}^{<} \epsilon^{(s)}\right)
$$

Considering that the turbulent field remains near equilibrium along mean streamlines, $D\left\langle k^{(s)}\right\rangle / D t=$ $D\left\langle\epsilon^{(s)}\right\rangle / D t=0$ leads to the two balance equations

$$
P^{(s)}=\epsilon^{(s)}-J_{k}^{(s+r)}\left(k^{(s)}\right)+\frac{D k^{(s)<}}{D t}
$$


and

$$
C_{\epsilon_{1}}^{(s)} P^{(s)}-C_{\epsilon_{2}}^{(s)} \epsilon^{(s)}=-\frac{k^{(s)}}{\epsilon^{(s)}}\left[J_{\epsilon}^{(s+r)}\left(\epsilon^{(s)}\right)-\frac{D \epsilon^{(s)<}}{D t}\right]
$$

still with the constraint $\left\langle D k^{(s)<} / D t\right\rangle=\left\langle D \epsilon^{(s)<} / D t\right\rangle=0$. It is again important to emphasize that the diffusion terms $J_{k}^{(r)}$ and $J_{\epsilon}^{(r)}$ (see appendix D)

$$
J_{k}^{(r)}\left(k^{(s)}\right)=-\frac{\partial}{\partial x_{j}}\left(u_{j}^{<} k^{(s)}\right) \quad \text { and } \quad J_{\epsilon}^{(r)}\left(\epsilon^{(s)}\right)=-\frac{\partial}{\partial x_{j}}\left(u_{j}^{<} \epsilon^{(s)}\right)
$$

are non zero and have also non zero mean values. Consequently, these terms cannot be neglected from a physical point of view. The analysis can be pursued. Now, eliminating $P^{(s)}$ between these two latter equations yields

$$
C_{\epsilon_{2}}^{(s)}-C_{\epsilon_{1}}^{(s)}=\frac{k^{(s)}}{\epsilon^{(s) 2}}\left[J_{\epsilon}^{(s+r)}\left(\epsilon^{(s)}\right)-\left(\frac{D \epsilon^{(s)<}}{D t}\right)\right]-\frac{C_{\epsilon_{1}}^{(s)}}{\epsilon^{(s)}}\left[J_{k}^{(s+r)}\left(k^{(s)}\right)-\left(\frac{D k^{(s)<}}{D t}\right)\right]
$$

The functional variation can be written formally in a compact form as

$$
\delta C_{\epsilon_{2}}^{(s)}=a_{1} \delta k^{(s)}+a_{2} \delta \epsilon^{(s)}+a_{3} \delta J_{k}^{(s+r)}+a_{4} \delta J_{\epsilon}^{(s+r)}+a_{5} \delta\left(\frac{D k^{(s)<}}{D t}\right)+a_{6} \delta\left(\frac{D \varepsilon^{(s)<}}{D t}\right)
$$

where $a_{i}=a_{i}\left(k^{(s)}, \epsilon^{(s)}\right)$ are some functions of $k^{(s)}$ and $\epsilon^{(s)}$ that are not detailed for sake of discussion. Each variation of the filtered variable denoted $\delta(\bar{\psi})$ appearing in the RHS of Eq. (93) can be developed as $\delta(\bar{\psi})=\delta(\langle\psi\rangle)+\delta\left(\psi^{<}\right)$composed as a statistical mean part variation $\delta(\langle\psi\rangle)$ and a large fluctuating part variation $\delta\left(\psi^{<}\right)$. Taking the statistical mean of Eq. (93) gives

$$
\left\langle\delta C_{\epsilon_{2}}^{(s)}\right\rangle=a_{1} \delta\left\langle k^{(s)}\right\rangle+a_{2} \delta\left\langle\epsilon^{(s)}\right\rangle+a_{3} \delta\left\langle J_{k}^{(s+r)}\right\rangle+a_{4} \delta\left\langle J_{\epsilon}^{(s+r)}\right\rangle
$$

At this step, it can be pointed out that $\delta C_{\epsilon_{2}}^{(s)}$ cannot be considered as fluctuating because it is a numerical parameter of the model so that $\left\langle\delta C_{\epsilon_{2}}^{(s)}\right\rangle=\delta C_{\epsilon_{2}}^{(s)}$. In this condition, Eq. (94) allows to recover the main results obtained in the preceding section 4.1 and especially Eq. (86). Subtracting Eq. (94) from Eq. (93) yields the resulting equation

$$
0=a_{1} \delta k^{(s)<}+a_{2} \delta \epsilon^{(s)<}+a_{3} \delta J_{k}^{(s+r)<}+a_{4} \delta J_{\epsilon}^{(s+r)<}+a_{5} \delta\left(\frac{D k^{(s)<}}{D t}\right)+a_{6} \delta\left(\frac{D \varepsilon^{(s)<}}{D t}\right)
$$

that governs the large scale fluctuations $k^{(s)<}$ and $\epsilon^{(s)<}$. This outcome clearly demonstrates that the variations of $\delta\left(k^{(s)}\right)$ and $\delta\left(\epsilon^{(s)}\right)$ possibly differ from $\delta\left(\left\langle k^{(s)}\right\rangle\right)$ and $\delta\left(\left\langle\epsilon^{(s)}\right\rangle\right)$ while Eq. (86) is still verified. 


\section{$5 \quad$ Physical underlying mechanisms}

The basic reason why we get a linear response to the variation of $C_{\epsilon_{2}}^{(s)}$ is the account of the additional diffusion contribution coming from the convective terms in the large scale instantaneous equations. This term must be non zero if we require consistency with the averaged equations, which is physically necessary. The instantaneous equations (29) and (44) respectively for the subfilter energy and for the dissipation rate, are resolved numerically and in particular their LHS corresponding to $\mathcal{D} k^{(s)} / \mathcal{D} t$ and $\mathcal{D} \varepsilon^{(s)} / \mathcal{D} t$ are resolved numerically. But we know physically that these terms have non zero mean value. For the purpose of the variational study, an analytical approximation is derived in appendix D which are indeed crude but have the merit to give the correct mean value which is in fact the diffusion by large scale motion as indicated by Eq. (170). Again, we insist on the fact that this term comes in addition to the small scales diffusion to sum up as a total diffusion involving the total eddy viscosity appearing in the key diffusion equation (73) rather than the subgrid eddy viscosity. As a result, Eq. (80) follows and explains this outcome.

\section{$6 \quad$ Cutoff with anisotropic filters}

For practical engineering flows in complex geometries with rigid walls and for geophysical flows encountered in nature, the grid resolution can be deformed with a high degree of anisotropy in the streamwise, spanwise and normal directions to the wall. In this situation, it is necessary to correctly estimate the function $r\left(\kappa_{c}\right)$ used in the coefficient $C_{\epsilon_{2}}^{(s)}\left[r\left(\kappa_{c}\right)\right]$ for performing numerical simulations. To do that, one has to determine fairly well the equivalent cutoff value that accounts for the anisotropy of the filter. This issue is of utmost importance because it will directly influence the value of the coefficient $C_{\epsilon_{2}}^{(s)}$. The filter is supposed to be determined by the three spatial dimensions $\Delta_{1}, \Delta_{2}, \Delta_{3}$ in the streamwise, spanwise and normal directions of the flows $x_{1}, x_{2}, x_{3}$ in physical space or $\kappa_{c_{1}}, \kappa_{c_{2}}, \kappa_{c_{3}}$ in spectral space. The formulae proposed by Deardorff [41] is often used in practice by many authors. This hypothesis express in fact the conservation of the integration elementary volume (in physical space or in spectral space as well). It is really valid only if the anisotropy is weak. Another formulae comes from the work of Bardina et al. [42] inspired from inertia moments. Let us recall these formulae. The hypothesis of Deardorff [41] is well suited for weakly anisotropic grids by considering an equivalent length-scale as 
$\Delta_{D f}=\left(\Delta_{1} \Delta_{2} \Delta_{3}\right)^{1 / 3}$ while the hypothesis of Bardina et al. [42] build for anisotropic grids is given by a quadratic approximation $\Delta_{B a}=\left[\left(\Delta_{1}^{2}+\Delta_{2}^{2}+\Delta_{3}^{2}\right) / 3\right]^{1 / 2}$ and gives preeminence to the largest components. In the case of high filter anisotropy, the Bardina et al. hypothesis roughly gives the maximum of $\Delta_{1}, \Delta_{2}, \Delta_{3}$ which is very different from the Deardorff hypothesis and can be physically plausible if one considers that turbulence energy is mainly concentrated in larger eddies. Recognizing this fact, the present authors Chaouat and Schiestel $[36,43]$ have used a combination of both hypotheses such as

$$
\Delta=\zeta \Delta_{D f}+(1-\zeta) \Delta_{B a}
$$

where $\zeta$ is an adjustable numerical parameter depending on the grid refinement used to tackle engineering flows in complex geometries. A new contribution was done by Scotti and Meneveau [44], as well as Scotti et al. [45] based on integration of spectral fluxes for use in the Smagorinsky model. On this purpose, one has to note that the adequate formulae depends not only of the geometrical properties of the filter but also of the type of function to be considered. The Deardorff hypothesis is better suited for almost constant functions while Bardina's is rather recommended for fast decaying spectra. To improve the previous formulation (96) used in the PITM model, the basic idea is to integrate the energy spectrum which is rapidly decaying if one refers to the Kolmogorov law. We chose here to adapt the Scotti and Meneveau [44] type of approach to the case of the energy spectrum rather than the Smagorinsky model noticing that the spectral decay laws for turbulence energy and for cascade transfer are very different. To do this, let us suppose $\kappa_{c_{1}}>\kappa_{c_{2}}>\kappa_{c_{3}}$ correspondingly in spectral space. Considering that only approximate relations are required, we shall not consider the precise mathematical form of the filter function but only the characteristic lengths in the three dimensions. In spectral space, the one-dimensional Kolmogorov spectrum reads

$$
E(\kappa)=C_{\kappa} \epsilon^{2 / 3} \kappa^{-5 / 3}
$$

while the corresponding three-dimensional spectrum is

$$
\boldsymbol{E}_{i s o}(\boldsymbol{\kappa})=\frac{C_{\kappa}}{4 \pi} \epsilon^{2 / 3}|\boldsymbol{\kappa}|^{-11 / 3}=\frac{C_{\kappa}}{4 \pi \kappa^{2}} \epsilon^{2 / 3} \kappa^{-5 / 3}
$$

At this step, we require that the integral under the anisotropic filter function be equal to the integral under a fictitious isotropic filter that defines the "equivalent "cutoff denoted $\kappa_{e}$. The spectral domain 
of integration for the wave vector $\boldsymbol{\kappa}$, associated to $\Delta_{1} \Delta_{2} \Delta_{3}$ in the physical space, can be decomposed, in a first approximation, into three separate domains such as for $\kappa \in\left[\kappa_{0}, \kappa_{1}\right], d \mathcal{V}(\kappa)=4 \pi \kappa^{2} d \kappa$; for $\kappa \in$ $\left[\kappa_{1}, \kappa_{2}\right], d \mathcal{V}(\kappa)=4 \pi \kappa \kappa_{1} d \kappa$; and for $\kappa \in\left[\kappa_{2}, \kappa_{3}\right], d \mathcal{V}(\kappa)=4 \kappa_{1} \kappa_{2} d \kappa$ where $\kappa_{0}$ stands for the arbitrary wave number at the beginning of the Kolmogorov energy spectrum. As we will see, this choice has however no effect on the final result. So, one can write with a good approximation

$$
\int_{\kappa_{0}}^{\kappa_{e}} E(\kappa) d \kappa \approx \int_{\kappa_{0}}^{\kappa_{1}} \boldsymbol{E}_{i s o}(\kappa) 4 \pi \kappa^{2} d \kappa+\int_{\kappa_{1}}^{\kappa_{2}} \boldsymbol{E}_{i s o}(\kappa) 4 \pi \kappa_{1} \kappa d \kappa+\int_{\kappa_{2}}^{\kappa_{3}} \boldsymbol{E}_{i s o}(\kappa) 4 \kappa_{1} \kappa_{2} d \kappa
$$

Then,

$$
\int_{\kappa_{0}}^{\kappa_{e}} \kappa^{-5 / 3} d \kappa \approx \int_{\kappa_{0}}^{\kappa_{1}} \kappa^{-5 / 3} d \kappa+\kappa_{1} \int_{\kappa_{1}}^{\kappa_{2}} \kappa^{-8 / 3} d \kappa+\frac{\kappa_{1} \kappa_{2}}{\pi} \int_{\kappa_{2}}^{\kappa_{3}} \kappa^{-11 / 3} d \kappa
$$

after straightforward calculation is leading to

$$
\kappa_{e} \approx\left[\frac{3}{5} \kappa_{1}^{-2 / 3}+\left(\frac{2}{5}-\frac{1}{8 \pi}\right) \kappa_{1} \kappa_{2}^{-5 / 3}+\frac{1}{4 \pi} \kappa_{1} \kappa_{2} \kappa_{3}^{-8 / 3}\right]^{-3 / 2}
$$

It is possible to express this result under an equivalent formulae by introducing the ratios $r_{2}=\kappa_{2} / \kappa_{1}$ and $r_{3}=\kappa_{3} / \kappa_{1}$, each greater than unity

$$
\frac{\kappa_{e}}{\kappa_{1}} \approx\left[\frac{3}{5}+\left(\frac{2}{5}-\frac{1}{8 \pi}\right) r_{2}^{-5 / 3}+\frac{1}{4 \pi} r_{2} r_{3}^{-8 / 3}\right]^{-3 / 2}
$$

or

$$
\frac{\kappa_{e}}{\kappa_{D f}}=\left[\frac{3}{5} r_{2}^{2 / 9} r_{3}^{2 / 9}+\left(\frac{2}{5}-\frac{1}{8 \pi}\right) r_{2}^{-13 / 9} r_{3}^{2 / 9}+\frac{1}{4 \pi} r_{2}^{11 / 9} r_{3}^{-22 / 9}\right]^{-3 / 2}
$$

where $\kappa_{D f}=\left(\kappa_{1} \kappa_{2} \kappa_{3}\right)^{1 / 3}$ is the wave number associated with the formulation of Deardorff [41]. In the particular case of the plane channel flow with $r_{2}=\kappa_{2} / \kappa_{1}$ near unity and $r_{3}=\kappa_{3} / \kappa_{1}$ large because of the grid refinement in the normal direction to the wall $x_{3}$, we get a much simpler formulae. This correspond to the case of "pancake", filters in physical space (i.e. pencil-like in spectral space)

$$
\kappa_{e}=\frac{\left(\kappa_{1}^{2} \kappa_{2}\right)^{1 / 3}}{\left(1-\frac{1}{8 \pi}\right)^{3 / 2}} \approx 1.06 \frac{\kappa_{D f}}{r_{3}^{1 / 3}}
$$

The same relations obviously hold for $\eta_{c}=L \kappa_{c}=\pi L / \Delta$. Thus only the smallest wavelength acts significantly while the largest wavelength has no influence. This is due to the fact that the area of the 
$\kappa_{c 1}<\kappa_{c 2}<\kappa_{c 3}$ ellipsoid far from the origin is narrow and the energy spectrum in these areas is small. Besides, it is also interesting to examine the spectral counterpart of the Bardina formulae. From the definition of $\Delta_{B a}$ one may infer

$$
\kappa_{B a}=\frac{\sqrt{3}}{\left(\frac{1}{\kappa_{1}^{2}}+\frac{1}{\kappa_{2}^{2}}+\frac{1}{\kappa_{3}^{2}}\right)^{1 / 2}}
$$

Simple algebra shows that equivalently

$$
\kappa_{B a}=\frac{\sqrt{3} \kappa_{D f}}{\left(r_{2}^{2 / 3} r_{3}^{2 / 3}+r_{2}^{-4 / 3} r_{3}^{2 / 3}+r_{2}^{2 / 3} r_{3}^{-4 / 3}\right)^{1 / 2}}
$$

For the plane channel with $r_{2}=\kappa_{2} / \kappa_{1} \approx 1$ and $r_{3}=\kappa_{3} / \kappa_{1}$ large, we get

$$
\kappa_{B a}=\sqrt{\frac{3}{2}}\left(\kappa_{1}^{2} \kappa_{2}\right)^{1 / 3} \approx 1.22 \frac{\kappa_{D f}}{r_{3}^{1 / 3}}
$$

Comparing Eq. (107) with Eq. (104) leads to very similar results $\kappa_{B a} \approx 1.15 \kappa_{e}$ This outcome brings support for the approximations used by Chaouat [30], Chaouat and Schiestel [23] when they performed the turbulent channel flow over periodic hills. In the very general case in which the Kolmogorov power law is not verified, such as in the production region of the energy spectrum, the resulting relation could be cast under the following form

$$
\frac{\kappa_{e}}{\kappa_{D f}} \approx f\left(r_{2}, r_{3}, r_{L}\right)
$$

where $r_{L}=\kappa_{\delta} / \kappa_{1}$ introducing the characteristic length scale of the flow $\delta$ with $\kappa_{\delta}=\pi / \delta$. The parameter $r_{L}=\Delta_{1} / \delta$ corresponds to the local mesh coarseness or refinement. This is suggesting empirical laws like in Eq. (96) with

$$
\zeta=\frac{\Delta_{1}}{\delta+\Delta_{1}}
$$

where $\Delta_{1}$ is the maximum step size of the grid and $\delta$ the half-distance between parallel planes or any characteristic length in more general wall flows. In this way, the Bardina formulae is approached for small $\Delta_{1}\left(\lim _{\zeta \rightarrow 0} \Delta(\zeta)=\Delta_{B a}\right)$ (i.e. large integration domain of the filter in spectral space) and the Deardorff one is recovered for large $\Delta_{1}\left(\lim _{\zeta \rightarrow 1} \Delta(\zeta)=\Delta_{D f}\right)$ (i.e. small integration domain of the filter in spectral space). Let us consider now, the characteristic length scales for the subfilter zone $L^{(s)}=\left\langle k^{(s)}\right\rangle^{3 / 2} / \epsilon$ 
and for the full turbulence field $L=k^{3 / 2} / \epsilon$, their ratio will be denoted $L^{+(s)}=\left(\left\langle k^{(s)}\right\rangle / k\right)^{3 / 2}$. From equation (7), we get

$$
L^{+(s)}=\frac{1}{\left(1+\beta \eta_{c}^{3}\right)^{1 / 3}}=\frac{\Delta}{\left(\Delta^{3}+\beta(\pi L)^{3}\right)^{1 / 3}}=\frac{C^{+} \Delta^{+}}{\left(1+\left(C^{+} \Delta^{+}\right)^{3}\right)^{1 / 3}}
$$

where $\Delta^{+}=\Delta / L$ and $C^{+}=\beta^{-1 / 3} / \pi=\left(3 C_{\kappa} / 2\right)^{3 / 2} / \pi$. We consider Eq. (96) with (109) to the case of the plane channel flow in which the grid is refined near the walls and thus $\rho_{2}=\Delta_{2} / \Delta_{1}$ is near unity while $\rho_{3}=\Delta_{3} / \Delta_{1}$ is very small. This implies $\Delta_{D f}=\Delta_{1} \rho_{3}^{1 / 3}$ and $\Delta_{B a}=\Delta_{1}\left[\left(2+\rho_{3}^{2}\right) / 3\right]^{1 / 2}$. From Eqs. (96) and (109), it is a simple matter to obtain

$$
\Delta\left(\rho_{3}\right)=\Delta_{1}\left[\frac{\Delta_{1}}{\delta+\Delta_{1}} \rho_{3}^{1 / 3}+\frac{\delta}{\delta+\Delta_{1}}\left(\frac{2+\rho_{3}^{2}}{3}\right)^{1 / 2}\right]
$$

Two cases are now studied. In the case of isotropic grids, $\rho_{3} \approx 1$ so that $\Delta \approx \Delta_{1}$, and the length-scale given by Eq. (110) is then given by

$$
\lim _{\rho_{3} \rightarrow 1} L^{+(s)}\left(\rho_{3}\right)=\frac{C^{+} \Delta_{1}^{+}}{\left(1+\left(C^{+} \Delta_{1}^{+}\right)^{3}\right)^{1 / 3}}
$$

where $\Delta_{1}^{+}=\Delta_{1} / L$. In the case of anisotropic grids, $\rho_{3} \approx 0$ so that $\Delta$ goes to

$$
\lim _{\rho_{3} \rightarrow 0} \Delta\left(\rho_{3}\right)=\left(\frac{2}{3}\right)^{1 / 2} \frac{\delta}{\delta+\Delta_{1}} \Delta_{1}
$$

and the length-scale is then given by

$$
\lim _{\rho_{3} \rightarrow 0} L^{+(s)}\left(\rho_{3}\right)=\frac{C^{+} \frac{\delta}{\delta+\Delta_{1}} \Delta_{1}^{+}}{\left[1+\left(C^{+} \frac{\delta}{\delta+\Delta_{1}} \Delta_{1}^{+}\right)^{3}\right]^{1 / 3}}
$$

For the limiting value when $\Delta_{1} \ll 1$, the function given by Eq. (112) goes to

$$
\lim _{\rho_{3} \rightarrow 1} L^{+(s)}\left[\Delta^{+}\left(\rho_{3}\right)\right]=C^{+} \Delta_{1}^{+}+O\left(\Delta_{1}^{+}\right)^{2}
$$

and the other one given by Eq. (114) goes to

$$
\lim _{\rho_{3} \rightarrow 0} L^{+(s)}\left[\Delta^{+}\left(\rho_{3}\right)\right]=\frac{\delta C^{+} \Delta_{1}^{+}}{\delta+\Delta_{1}}+O\left(\Delta_{1}^{+}\right)^{2}
$$

Obviously, both functions go to unity when $\Delta_{1}^{+}$goes to infinity. Figure 3 shows the evolution of these functions given by Eqs. (112) and (114), respectively versus the ratio $\Delta_{1}^{+}=\Delta_{1} / L$. Relatively to 
the case of an isotropic filter, it can be seen that the curve associated with the case of an anisotropic filter is shifted to the right. These evolutions (see Fig. 1 in Ref. [32] for the purposes of comparison) show the difference between $L^{+2 / 3}$ and $L^{+2}$ distributions as a function of normalized filter width. It appears that the present $L^{+}$variation looks very similar but for a different reason. The present analysis

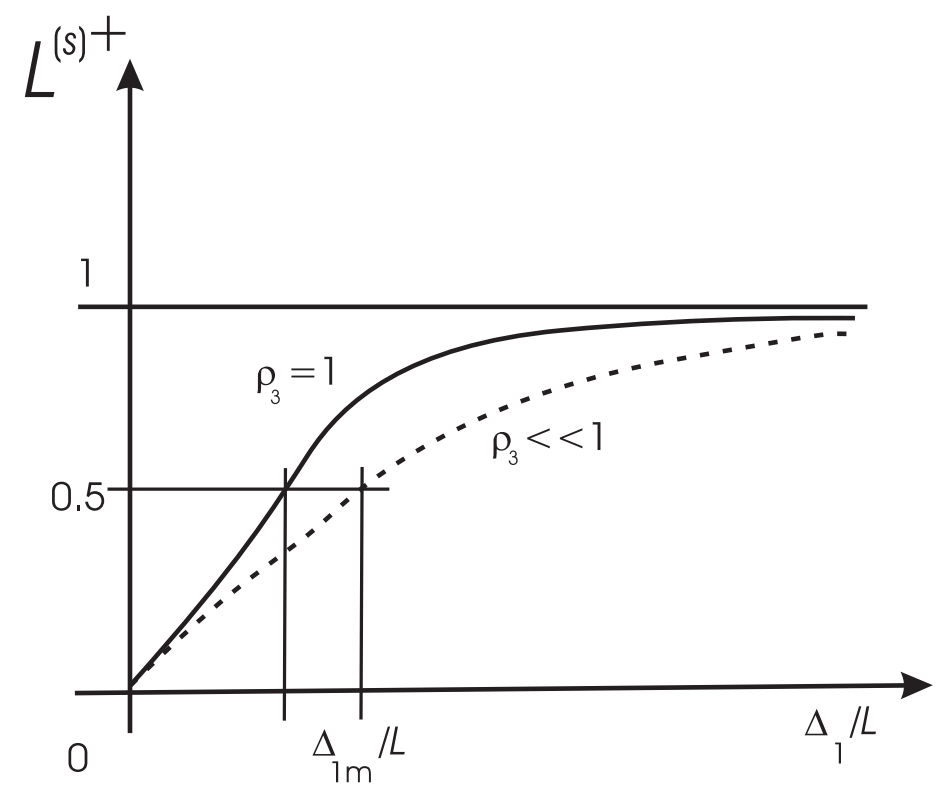

Figure 3: Variation of $L^{+(s)}$ given by Eqs. (112) and (114), respectively, versus the grid step $\Delta_{1}^{+}=\Delta_{1} / L$ for isotropic and anisotropic filters as functions of $\rho_{3}$

also shows that the sole use of Deardorff approximation of anisotropic meshes for the calculation of partial energies is therefore inadequate. The effective grid size is hugely underestimated, that is to say that the equivalent cutoff wavenumber is greatly overestimated and consequently, the partial subfilter energy would be also underestimated. In practice, the use of combined forms of Deardorff and Bardina hypotheses proved to be satisfactory and entirely supported by the integral analysis that has been conducted in this section. We had shown in the previous section that theoretical result $R=r$ remains valid in non-homogeneous flows. However, accounting of the anisotropy of the filter function influences appreciably the calculation results because the analytical relation of $R$ versus the grid step is modified. An incorrect account of filter anisotropy may corrupt the numerical results. For instance using the pure formulae proposed by Deardorff [41] in the PITM simulation for wall flows as it was done by Heinz et 
al. [33] may be questionable.

\section{$7 \quad$ Grid density considerations}

\subsection{Isotropic grids}

It can be of interest to get an estimate of the number of grid points $N$ first assuming locally a constant grid size $\Delta$ for PITM simulations. Depending of the cutoff wave number $\kappa_{c}=\pi / \Delta, N \propto V / \Delta^{3}$ where $V$ is the computational domain volume. This expression can be rewritten as $N=V \eta_{c}^{3} /(\pi L)^{3}$. The Kolmogorov law implies $\left\langle k^{(s)}\right\rangle / k=\left(3 C_{\kappa} / 2\right) \eta_{c}^{-2 / 3}$. Using the definition of the length-scale $L=k^{3 / 2} / \epsilon$, it is found that

$$
\frac{N}{V}=\left[\frac{1}{\pi}\left(\frac{3 C_{\kappa}}{2}\right)^{\frac{3}{2}}\right]^{3} \frac{1}{\left(L^{(s)}\right)^{3}}=\left(\frac{C^{+}}{L^{(s)}}\right)^{3}
$$

Equation (117) indicates that the mesh density varies according to the law $\left(L^{(s)}\right)^{-3}$ and not as $\left(L^{(s)}\right)^{-9}$ at it was erroneously asserted by Heinz [32]. Obviously, the number of grid points $N$ increases when the subfilter energy decreases because more large scales must be explicitly calculated by the numerical scheme. Conversely, when the subgrid energy increases, $N$ decreases because less large scales are computed since more subgrid scales are modeled, $\mathrm{N}$ is then going to its RANS value. The relation (117) is in fact similar to the classical result obtained in usual LES calculations with a cutoff in the inertial zone.

\subsection{Anisotropic grids}

The case of anisotropic filters is now illustrated in the case of the turbulent flow between two parallel plates. The number of grid points can be estimated from $N \propto V /\left(\Delta_{1} \Delta_{2} \Delta_{3}\right)$ that is to say $N \propto V / \Delta_{D f}^{3}$ regardless of the effective characteristic filter width chosen in the calculation. This latter will be something like equation (111) rewritten as

$$
\frac{\Delta}{\Delta_{D f}}=\zeta+(1-\zeta) \frac{\Delta_{B a}}{\Delta_{D f}}
$$


and taking into account the previous results,

$$
\frac{\Delta}{\Delta_{D f}}=\zeta+(1-\zeta)\left(\frac{2+\rho_{3}^{2}}{3 \rho_{3}^{2 / 3}}\right)^{1 / 2}
$$

The final result being

$$
\frac{N}{V}=\left(\frac{C^{+}}{L^{(s)}}\right)^{3}\left[\zeta+(1-\zeta)\left(\frac{2+\rho_{3}^{2}}{3 \rho_{3}^{2 / 3}}\right)^{1 / 2}\right]^{3}
$$

The estimates given by equations (117) and (120) are based upon physical quantities like $L^{(s)}$, and consequently they effectively correspond to the mesh size necessary for a meaningful simulation without being neither oversized nor undersized.

\section{DNS and PITM simulations}

\subsection{Illustration for a basic non-homogeneous flow, the plane channel}

In order to confirm the law $R(r)=r$ in the PITM model, we consider an application to the turbulent plane channel for differing values of the filter width. The balance of the terms involved in the mean epsilon equation is also illustrated. Indeed, the turbulent channel flow is a typical non-homogeneous flow confined between rigid walls in which like in the theoretical logarithmic layer, the turbulent diffusion terms are of primary importance in the epsilon equation balance. PITM numerical simulations are performed for the Reynolds number $R_{\tau}=u_{\tau} \delta / \nu=395$ where $u_{\tau}$ denotes the friction velocity and $\delta$ is the half channel width. The present results are then compared with reference DNS [46].

\subsection{Numerical PITM procedure}

The dimension of the channel in the streamwise, spanwise and normal directions along the axes $x_{1}, x_{2}$, $x_{3}$ are $L_{1}=6.4 \delta, L_{2}=3.2 \delta$ and $L_{3}=2 \delta$. For the Reynolds number studied here, the coarse and medium grids resolution are $84 \times 42 \times 84$ and $84 \times 42 \times 128$ in the $\left(x_{1}, x_{2}, x_{3}\right)$ directions. The mesh is uniform in the streamwise and spanwise directions $\left(x_{1}, x_{2}\right)$ and the grid spacings in wall unit are $\Delta_{1}^{+}=\Delta_{2}^{+}=30$, respectively. In the normal direction to the wall $x_{3}$, the grid points are distributed in different spacings 
with a refinement near the wall to compute accurately the velocity boundary layer according to the transformation $x_{3 j}=\tanh \left[\xi_{j} F\left(\xi_{j}\right)\right.$ atanh $\left.a\right] / 2$ where $\xi_{j}=-1+2(j-1) /\left(N_{3}-1\right)\left(j=1,2, \cdots N_{3}\right)$, $F\left(\xi_{j}\right)=\sqrt{\left(1+\xi_{j}^{2}\right) / 2}$, the parameter $a$ is a coefficient set to 0.990 for $N_{3}=84$ and 0.9875 for the $N_{3}=$ 128 leading to $\Delta_{3}^{+}=0.2$. For both cases, it is found that the larger grid spacing in the normal direction is then smaller than 15 in wall unit $\Delta_{3}^{+}<15$. The simulations are performed using the numerical code developed by Chaouat [47] which is based on the finite volume technique and optimized with message passing interface (MPI). The equations are integrated in time by means of an implicit Runge-Kutta scheme of fourth-order accuracy in time. The Navier-Stokes equations are solved in space by a centered scheme of fourth-order accuracy in space whereas the transport equations of turbulence are solved by an upwind scheme of second-order accuracy in space. The PITM model used to perform the simulation is based in the present case on a second moment-closure (SMC) that allows a refined description of the flow anisotropy thanks to the pressure-strain correlation term that redistributes the turbulence energy among the stress componants. Its formulation is described in detail in several references [11, 23, 35]. Moreover, a relaxation mechanism based on a return to equilibrium is superimposed to the coefficient $C_{\epsilon_{2}}^{(s)}$ during the computations. In practice it brings the model to relax more rapidly towards the target equilibrium energy ratio $r=\left\langle k^{(s)}\right\rangle / k$. This term is has a clear physical meaning and has been used in spectral models by Yassour and Wolfshtein [48]. The coefficient $C_{\epsilon_{2}}^{(s)}$ is then dynamically modified as $C_{\epsilon_{2}}^{(s)}+\Delta C_{\epsilon_{2}}^{(s)}$ by means of the parameter $r$ computed in the simulation itself in time and space with

$$
\Delta C_{\epsilon_{2}}^{(s)}=\chi C_{\epsilon_{2}}^{(s)}\left(1-\frac{r}{r_{e q}}\right)
$$

The target value $r_{e q}$ is given by Eq. (7) and $\chi$ is a constant parameter empirically estimated by $\chi \simeq 0.2$. The mechanisms of this process are fully described by Chaouat and Schiestel [49]. Considering that we have to deal with statistical values, the energy ratio $r$ appearing in Eq. (121) is averaged in time but also in space in the homogeneous directions of the flow as far as possible. Space averaging can easily been made at each time step but time averaging on short periods is made on the basis of a simple recursive procedure as indicated by Chaouat [47]. The turbulence length-scale $L=k^{3 / 2} / \epsilon$ used for computing the parameter $\eta_{c}=\kappa_{c} L$ is also averaged in time using the same procedure. On the numerical point of view, this procedure is particularly useful for stabilizing the calculation when the large scale motions of the flow vary rapidly both in time and space. It is also justified not only from a numerical point of view but also from a physical point of view, because as stated before, these relations are supposed 
to be statistical relations and thus need averaging to be meaningful. As it could be mentioned, the simulation performed using the PITM method requires this specific procedure because of the coupling arising from the coefficient $C_{\epsilon_{2}}^{(s)}$ and the energy ratio $r$, contrarily to the PANS method that considers a constant ratio. The authors would like to state that this is the key concept to get a dynamic model that is able to see the grid step of the mesh like in large eddy simulations. In the application to the turbulent plane channel flow, a constant pressure gradient term is included in the momentum equation to balance the viscous effects at the walls aiming to get a periodic condition between the inlet and outlet plane sections of the channel. The boundary conditions imposed at the lower and upper walls located at $x_{3}=0$ and $2 \delta$ of the channel are no slip velocity conditions $\bar{u}_{i}=0$.

\subsection{Numerical DNS procedure}

The DNS simulation performed by Chaouat and Peyret [46] on a very refined mesh for the same Reynolds number $R_{\tau}=395$ is considered in the following for computing the ratio $r_{D N S}=\left\langle k^{(s)}\right\rangle / k$. The dimension of the channel is identical as the one chosen for the PITM simulation, $L_{1}=6.4 \delta, L_{2}=3.2 \delta$ and $L_{3}=2 \delta$ and the equations were integrated in time using an explicit Runge-Kutta scheme of fourth order accuracy in time and solved in space by means of a centered scheme of fourth order accuracy in space. The DNS grid resolution in the $\left(x_{1}, x_{2}, x_{3}\right)$ directions is $1024 \times 512 \times 512$ and the spacings in wall unit are very small $\Delta_{1}^{+}=\Delta_{2}^{+} \simeq 2.5$ with $\Delta_{3}^{+}<2$. The filtered variable $\bar{\phi}$ on the coarse grid is in principle determined by a filtering operation as the convolution with a filter $G$ in space $\bar{\phi}=G * \phi$ defined as $[36,35]$

$$
\bar{\phi}(\boldsymbol{x}, t)=\int_{\mathcal{D}} G[\boldsymbol{x}-\boldsymbol{\xi}, \Delta(\boldsymbol{x}, t)] \phi(\boldsymbol{\xi}, t) d \boldsymbol{\xi}
$$

where in this expression, $\Delta$ denotes the filter-width that varies in time and space and $\mathcal{D}$ denotes the infinite flow domain. In practice for discrete solution, the filtered variable $\bar{\phi}$ in the $x_{i}$ direction at the grid-point $\left(x_{i}\right)_{j}$ is computed by integrating the DNS variable $\phi$ over the cell $\Omega$ by means of an explicit discrete approximation to the top hat filter defined as

$$
\bar{\phi}\left(x_{i}\right)_{j}=G(\phi)\left(\Delta,\left(x_{i}\right)_{j}\right)=\sum_{m=-n_{j}}^{m=l_{j}}\left(\alpha_{i}\right)_{j m} G\left[\left(x_{i}\right)_{j+m}-\left(x_{i}\right)_{j}, \Delta\left(x_{i}\right)_{j}\right] \phi\left[\left(x_{i}\right)_{j+m}\right]
$$


or in a more practical way,

$$
\bar{\phi}\left(x_{i}\right)_{j}=\sum_{m=-n_{j}}^{m=l_{j}}\left(a_{i}\right)_{j m}\left(\phi_{i}\right)_{j+m}
$$

where $\left(\phi_{i}\right)_{j+m}=\phi\left[\left(x_{i}\right)_{j+m}\right],\left(a_{i}\right)_{j m}=\left(\alpha_{i}\right)_{j m} G\left[\left(x_{i}\right)_{j+m}-\left(x_{i}\right)_{j}, \Delta\left(x_{i}\right)_{j}\right]$ are some numerical coefficients and $n_{j}, l_{j}$ are integer variables $\in N$ used in the determination of the interval $\Delta\left[\left(x_{i}\right)_{j}\right]$. The numerical integration covers a sufficiently wide interval

$$
I\left(x_{i}\right)_{j}=\left[\left(x_{i}\right)_{j-n_{j}},\left(x_{i}\right)_{j+l_{j}}\right]
$$

and is characterized by integration weights $\left(\alpha_{i}\right)_{j m}$. These integration weights are given by the quadrature rule that is selected such as the composite trapezoidal rule or Simpson integration [51]. The trapezoidal rule of integration is applied here because the spacings $\left(\Delta_{i}\right)_{j}^{+}=\left[\left(x_{i}^{+}\right)_{j}-\left(x_{i}^{+}\right)_{j+1}\right]$ in wall unit of the DNS grid are very small $\left(\left(\Delta_{i}\right)_{j}^{+}<2.5\right)$, so that it is sufficiently accurate. The coefficient used in the integration coincides with the spectral method of a flat low pass filter $G_{3}$ for three point formulae $[35,50]$. This procedure is then applied for the velocity $\phi=u_{i}$ in all $i t h$ directions and the filter-width corresponding to the interval $I\left(x_{i}\right)_{j}$ computed as $\Delta\left[\left(x_{i}\right)_{j}\right]=\left|\left(x_{i}\right)_{j+l_{j}}-\left(x_{i}\right)_{j-n_{j}}\right|$ is taken as the grid size of the cell $\Omega$ of the coarse PITM mesh $84 \times 42 \times 128$. The resolved scale turbulent energy is then computed by the relation

$$
k^{(r)}=\frac{1}{2}\left(\bar{u}_{i} \bar{u}_{i}-\left\langle u_{i}\right\rangle\left\langle u_{i}\right\rangle\right)
$$

The averaged resolved energy in space $\left\langle k^{(r)}\right\rangle$ is given by taking the averaging in the homogeneous directions leading to

$$
\left\langle k^{(r)}\right\rangle=\frac{1}{2}\left(\left\langle\bar{u}_{i} \bar{u}_{i}\right\rangle-\left\langle u_{i}\right\rangle\left\langle u_{i}\right\rangle\right)
$$

The subfilter scale turbulence energy is finally computed as the difference between the turbulent energy $k$ given by the DNS and the averaged resolved turbulent energy $\left\langle k^{(r)}\right\rangle$ given by Eq. (127) as

$$
\left\langle k^{(s)}\right\rangle=k-\left\langle k^{(r)}\right\rangle
$$

The objective is then to compute the ratio $r_{D N S}=\left\langle k^{(s)}\right\rangle / k$ obtained from the DNS simulation [46] and to compare its evolution with the PITM ratio value $r_{P I T M}$ returned by the simulation. 


\subsection{Numerical results}

Figures 4-5 illustrate the PITM numerical simulation. Figure 4 exhibits the ratio $\Delta / \Delta_{D f}$ versus the dimensionless wall distance for different values of the parameter $\zeta$ introduced in Eq. (96) and for the medium grid $84 \times 42 \times 128$. This ratio measuring the degree of the grid anisotropy is relatively high near the wall of around 3.5 and decreases regularly to unity when going away from the wall because the grids become more isotropic. As the cutoff wave number is computed using the filter width $\kappa_{c}=\pi / \Delta$, the ratio of the subgrid energy to the total energy is then affected by the calculation of the grid. In particular, the subgrid energy contribution remains of higher intensity for anisotropic grids than for isotropic grids. This figure simply confirms that the accounting for anisotropic grids in the wall region is essential in PITM simulations. In practice the numerical value $\zeta \simeq 0.5$ proved to be satisfactory. Figure 5 displays the profile of the ratio of the turbulence length-scale $L^{+(s)}=L^{(s)} / L$ versus the dimensionless wall distance returned both by the simulation and by Eq. (110) deduced from Eq. (7). Except in the very near wall region where a discrepancy is observed between the computed and the analytical results, probably due to the fact that the model does not work in a purely RANS mode as expected for the present mesh, both profiles shows similar evolutions marked by a regular decrease. Figure 6 illustrates the DNS simulation, it exhibits the evolution of the numerical ratio $r_{D N S}=\left\langle k^{(s)}\right\rangle / k$ obtained by filtering the DNS velocity field from the very fine DNS grid $1024 \times 512 \times 512$ onto the PITM coarse grid $84 \times 42 \times 128$ as well as the PITM ratio $r_{P I T M}$ returned by the simulation. As a result of interest, it is found that an excellent agreement is obtained in the logarithmic near wall region but small discrepancies appear both far away from the wall and in the wall sublayer region. Indeed, the two curves are very close together in the logarithmic wall region and afterwards slightly deviate from each over when moving to the center of the channel. This result shows that the PITM method returns a modeled energy $\left\langle k^{(s)}\right\rangle$ that is consistent with the coarse grid used in the simulation of the channel flow studied here. In that sense, it is a good achievement that the partially integrated transport modeling gives such a degree of accuracy in the near equilibrium wall region. The discrepancy observed in the center of the channel may arise from the cross correlations between the small scales and large scales that have been omitted in practice (see Appendix A). In the immediate vicinity of the wall, the low Reynolds effects may obscure the physical behavior. We shall thus remember the good agreement in the equilibrium layer which is the main interest of the present study. If these DNS results cannot constitute 


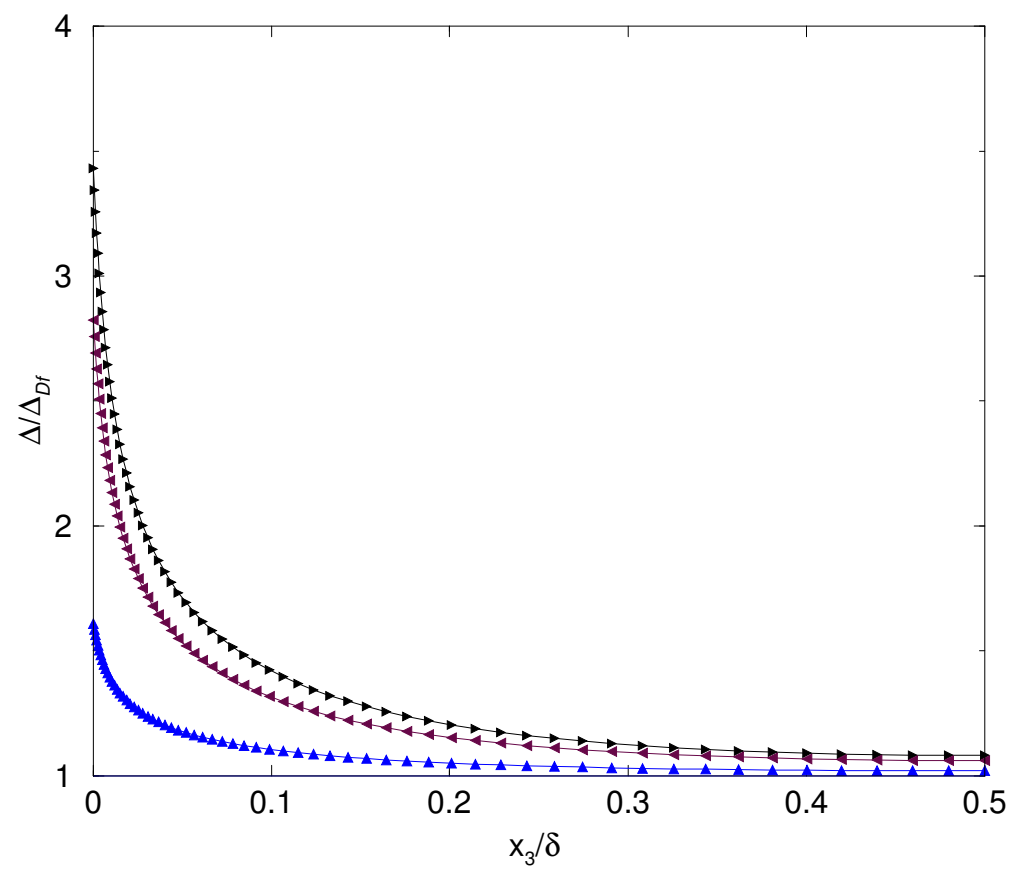

Figure 4: Variation of the ratio $\Delta / \Delta_{D f}$ versus the dimensionless wall distance for different values of the parameter $\zeta$ introduced in Eq. (96). $\zeta=0.25: \nabla ; \zeta=0.50: \triangleleft ; \zeta=0.75: \boldsymbol{\Delta} ; \zeta=1: \Delta / \Delta_{D f}=1$. Medium grid $84 \times 42 \times 128$

a mathematical proof, they however support the correct behavior of the subfilter model. Figure 7 shows the plots of the ratio $R(r)=\left(C_{\epsilon_{2}}^{(s)}-C_{\epsilon_{1}}^{(s)}\right) /\left(C_{\epsilon_{2}}-C_{\epsilon_{1}}\right)$ determined analytically in the PITM model versus the calculated ratio $r=\left\langle k^{(s)}\right\rangle / k$ returned by the PITM numerical simulation, using points located in the direction normal to the wall. The results are obtained from the PITM simulations performed on the coarse and medium grids. One can see that this ratio evolves linearly as expected, except in the immediate vicinity of the wall where the ratio $\left\langle k^{(s)}\right\rangle / k$ takes on its maximum values. The deviation from the linear law is due to the fact that the computed ratio $\left\langle k^{(s)}\right\rangle / k$ returned by the simulation is not equal to the target equilibrium value given by Eq. (7), as explained before. Consequently, the truly linear profile can be achieved only in equilibrium regions such as the logarithmic region. This figure clearly supports the behavior $R=r$ for the model in non-homogeneous flows (as well as homogeneous flows) in the near equilibrium region. As already observed in Fig. 6, this one also suggests that 


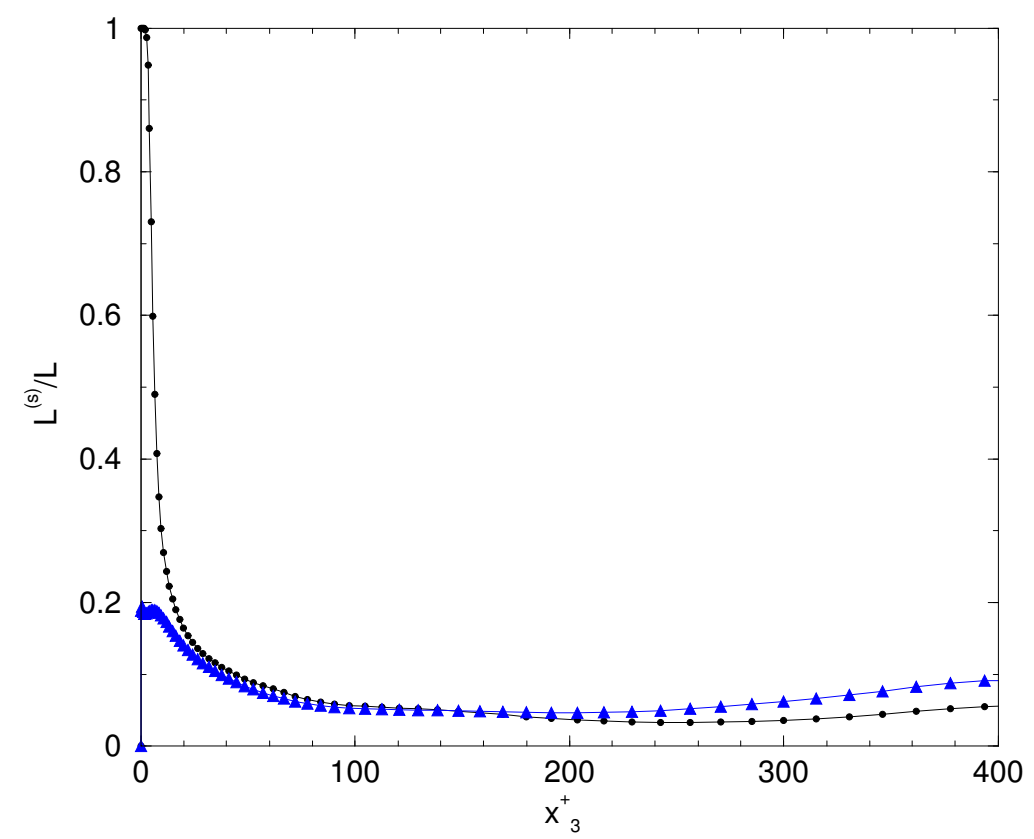

Figure 5: Variation of the ratio of the turbulence length-scale $L^{+(s)}=L^{(s)} / L$ versus the dimensionless wall distance. Analytical profile computed by Eq. (110) : •. Numerical profile : $\Delta$. Medium grid $84 \times 42 \times 128$

roughly one-third of energy is modeled while two-third of energy is explicitly simulated in the present case. This is merely a consequence of the particular choice of grid point distribution. We underline again that the ratio $r$ does not necessarily reach its target value $r_{e q}$ in the mechanism associated with Eq.(121) in zones of the flow that may be out of spectral equilibrium $[52,53,54]$. This result is in perfect agreement with the computational ratio value $r_{D N S}$ plotted in Fig. 6 providing an additional favorable behavior concerning the spectral distribution of energy. As the turbulent channel flow has been deeply investigated in previous papers by the authors themselves using different variants of the subfilter scale stress model [11, 20,43], only few results of the simulation performed recently by Chaouat [35] are recalled to serve as a discussion for the sake of consistency. In this latter reference paper mainly devoted to the commutation errors, numerical channel flow simulations were carried out on several grids including a sudden grid step increase in the grid-size in the streamwise direction. Some figures in this reference are discussed here to serve as illustration of the model capabilities related to the connection 


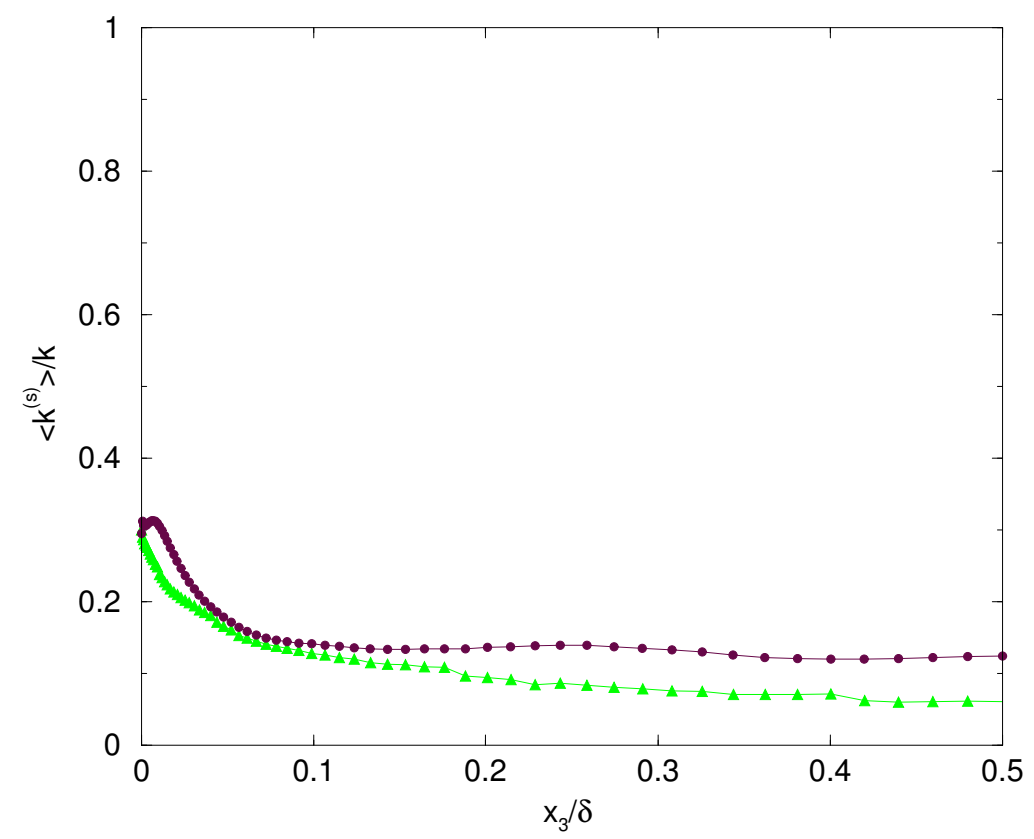

Figure 6: Computation of the numerical ratio $r_{D N S}=\left\langle k^{(s)}\right\rangle / k$ using the DNS data [46] associated with the refined grid $1024 \times 512 \times 512$ in comparison with the PITM ratio $r_{P I T M}$ computed on the coarse grid $84 \times 42 \times 128$. The filtered velocity fields are performed using the filtering operator $G$ defined in Eq. (124) applied on the DNS velocity fields. $r_{P I T M}: \bullet ; r_{D N S}: \Delta$

between grid and partial subfilter energy. The turbulence intensities associated with the subfilter scale stresses $\left\langle\tau_{i i}^{(s)}\right\rangle$ and the large scales stresses $\left\langle\tau_{i i}^{(r)}\right\rangle$ in the plane section of the channel versus the wall coordinate has revealed that the core flow is governed by the large scales of the flow whereas the wall flow region is dominated by the subfilter scales marked by the presence of the peaks of turbulence in the immediate vicinity of the wall (see Fig. 4 in Ref. [35]). The profiles of the streamwise, spanwise and normal turbulence intensities computed as the sum of the subgrid and resolved scale contributions has indicated a good overall agreement with the DNS data [35] (see Fig. 5 in Ref. [35]). At least, it can be noted that the sharing out of the turbulent energy among the subgrid and resolved contribution was studied in detail. It has been found that when the grid-size is modified (for instance increased), then a part of the energy contained into the resolved scales is removed and fed into the modeled scale but as expected, the sum of the modeled and resolved energy contribution remains almost the same. 


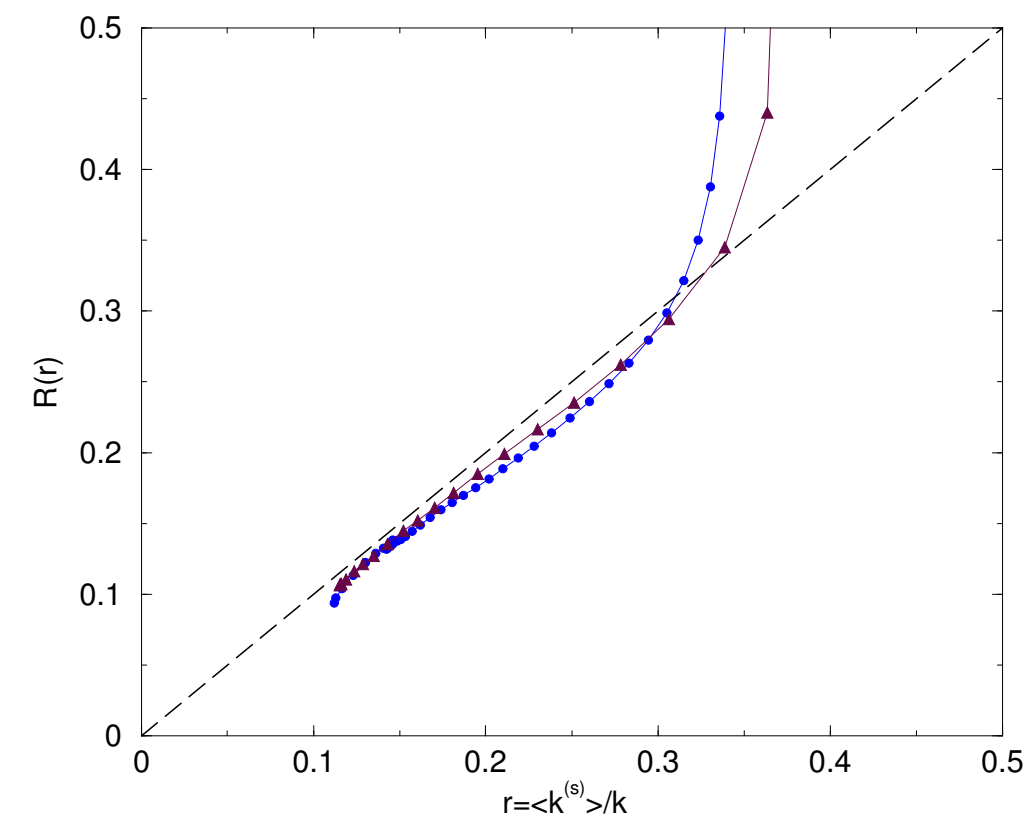

Figure 7: Variation of the analytical ratio $R(r)=\left(C_{\epsilon_{2}}^{(s)}-C_{\epsilon_{1}}^{(s)}\right) /\left(C_{\epsilon_{2}}-C_{\epsilon_{1}}\right)$ given by equation (8) versus the numerical ratio $r=\left\langle k^{(s)}\right\rangle / k$ computed from the simulation at points in the normal direction to the wall. Coarse grid $84 \times 42 \times 84: \Delta$. Medium grid $84 \times 42 \times 128$ :

Hence, it has been proved that the PITM model satisfies some principle of the energy conservation through the cutoff. The numerical results presented before are not intended to produce any proof of the linear behavior of the control function but it can be considered as a theoretical verification of the overall consistency of the previous results established from the variational analysis.

\section{Conclusion}

An heuristic study of the control mechanisms acting in the PITM hybrid models in response to variations in subfilter width has been carried on. It has been shown that the control function in the dissipation-rate transport equation in the PITM framework remains valid both in homogeneous turbulence and also as expected in non-homogeneous turbulence. In particular, the analysis based on variational calculus has 
been worked out and allowed to recover the exact linear dependence of the dissipation-rate coefficient with the partial energy ratio. This outcome put in light some functional aspects of the method in perfect agreement with the original PITM derivation. In addition, the problem of the cutoff wavenumber which characterizes the grid step size has been analyzed in detail and a practical formulae has been recommended to deal with anisotropic grids. To illustrate this point, the fully developed turbulent channel flow has been performed on several grids with respect to different filter widths and DNS data have been used to compute the ratio $\left\langle k^{(s)}\right\rangle / k$. As expected, this application confirms that the linear relation $R(r)=r$ is well verified according to the mathematical framework put in place. Satisfactory results for the subfilter energies were obtained, provided a well suited account of filter anisotropy is made. The present work should be helpful for users involved in hybrid RANS/LES methods and particularly the PITM method not only from a theoretical point of view but also in a practical point of view for simulating turbulent flows on relatively coarse grids with a drastic reduction of computational ressources.

\section{A Mathematical properties}

Let us consider

$$
\tau(f, g)=\overline{f g}-\bar{f} \bar{g}
$$

and

$$
\tau(f, g, h)=\overline{f g h}-\bar{f} \tau(g, h)-\bar{g} \tau(h, f)-\bar{h} \tau(f, g)-\bar{f} \bar{g} \bar{h}
$$

Eq. (129) can be developed in the following form as

$$
\tau(f, g)=\bar{f} \bar{g}-\bar{f} \bar{g}+\bar{f} g^{>}+\overline{\bar{g} f^{>}}+\overline{f^{>} g^{>}}
$$

It is then possible to get the averaging in statistical sense of (131) as

$$
\langle\tau(f, g)\rangle=\langle\overline{\bar{f}} \bar{g}\rangle-\langle\bar{f} \bar{g}\rangle+\left\langle\overline{\bar{f}} g^{>}\right\rangle+\langle\overline{\bar{g} f>}\rangle+\left\langle\overline{f^{>} g^{>}}\right\rangle
$$

Strictly speaking, the filtering process leads to mathematical non-equality $\overline{\langle\phi\rangle}=\langle\bar{\phi}\rangle \neq\langle\phi\rangle$ for any variable $\phi$. To recover $\langle\bar{\phi}\rangle=\langle\phi\rangle$, the key concept is to consider the tangent homogeneous anisotropic turbulence field at the physical space location implying that the variation of the mean varibale $\phi$ is 
accounted for by the use of Taylor series expansion in space limited to the linear terms [22, 36, 35]. In practice, $\langle\bar{\phi}\rangle \approx\langle\phi\rangle$ only if the variation of the flow velocities over the filter width is not too large. In the framework of the tangent homogeneous space and the spectral cutoff filter, $\overline{\langle\phi\rangle}=\langle\bar{\phi}\rangle=\langle\phi\rangle$ and $\left\langle\phi^{>} \phi^{<}\right\rangle=0$, so that Eq. (132) reduces to

$$
\langle\tau(f, g)\rangle=\left\langle f^{>} g^{>}\right\rangle
$$

and we get also

$$
\langle\tau(f, g, h)\rangle=\left\langle f^{>} g^{>} h^{>}\right\rangle
$$

In particular, the subfilter scale stress tensor defined in large eddy simulation is then

$$
\tau_{i j}^{(s)}=\tau\left(u_{i}, u_{j}\right)=\overline{u_{i} u_{j}}-\bar{u}_{i} \bar{u}_{j}
$$

while the resolved scale stress tensor is

$$
\tau_{i j}^{(r)}=\bar{u}_{i} \bar{u}_{j}-\left\langle u_{i}\right\rangle\left\langle u_{j}\right\rangle
$$

so that

$$
\tau_{i j}^{(s+r)}=\tau_{i j}^{(s)}+\tau_{i j}^{(r)}=\overline{u_{i} u_{j}}-\left\langle u_{i}\right\rangle\left\langle u_{j}\right\rangle
$$

In RANS modeling, the Reynolds stress tensor accounting for the total fluctuating velocities is defined as

$$
R_{i j}=\left\langle u_{i} u_{j}\right\rangle-\left\langle u_{i}\right\rangle\left\langle u_{j}\right\rangle
$$

Using the decomposition $\phi=\langle\phi\rangle+\phi^{<}+\phi^{>}$, the Reynolds stress tensor $R_{i j}$ can be rewritten as

$$
R_{i j}=\left\langle u_{i}^{<} u_{j}^{<}\right\rangle+\left\langle u_{i}^{>} u_{j}^{>}\right\rangle+\left\langle u_{i}^{<} u_{j}^{>}\right\rangle+\left\langle u_{i}^{>} u_{j}^{<}\right\rangle
$$

So that, assuming that the correlation between the small scale and large scale $\left\langle u_{i}^{<} u_{j}^{>}\right\rangle$are small compared to the other correlations, $R_{i j}$ can be computed in a first approximation as the sum of the statistical average of subfilter and resolved stresses

$$
R_{i j} \approx\left\langle\tau_{i j}^{(s+r)}\right\rangle=\left\langle\tau_{i j}^{(s)}\right\rangle+\left\langle\tau_{i j}^{(r)}\right\rangle
$$

and the contraction of the tensors appearing in Eq. (140) leads to

$$
k \approx\left\langle k^{(s+r)}\right\rangle=\left\langle k^{(s)}\right\rangle+\left\langle k^{(r)}\right\rangle
$$

where $k^{(s)}$ and $k^{(r)}$ are the filtered subfilter and resolved turbulence energy, respectively. This relation is strictly true in the case of the spectral cutoff filter, and only approximate in the case of a sharp filter. 


\section{B Formal derivative operators}

For sake of clarity, we consider in this section the use of a uniform spectral cutoff filter [22, 36, 35]. As recalled in Appendix A, each variable $\phi$ can be decomposed into a statistical mean $\langle\phi\rangle$, a macro-scale fluctuation $\phi^{<}$and a micro-scale fluctuation $\phi^{>}$. Using this decomposition for the velocity, it is a simple matter to see that the mass conservation implies

$$
\frac{\partial u_{j}^{<}}{\partial x_{j}}+\frac{\partial u_{j}^{>}}{\partial x_{j}}=0
$$

But the spectral cutoff filter also yields

$$
\frac{\partial u_{j}^{<}}{\partial x_{j}}=\frac{\partial u_{j}^{>}}{\partial x_{j}}=0
$$

The particle derivative in the instantaneous flow is given by

$$
\frac{d \phi}{d t}=\frac{\partial \phi}{\partial t}+u_{j} \frac{\partial \phi}{\partial x_{j}}
$$

The material derivative accounting for the statistical velocity is

$$
\frac{D \phi}{D t}=\frac{\partial \phi}{\partial t}+\left\langle u_{j}\right\rangle \frac{\partial \phi}{\partial x_{j}}
$$

and in particular

$$
\left\langle\frac{D \phi}{D t}\right\rangle=\frac{D\langle\phi\rangle}{D t}
$$

The material derivative accounting for the filtered velocity is

$$
\frac{\mathcal{D} \phi}{\mathcal{D} t}=\frac{\partial \phi}{\partial t}+\bar{u}_{j} \frac{\partial \phi}{\partial x_{j}}=\frac{\partial \phi}{\partial t}+\left\langle u_{j}\right\rangle \frac{\partial \phi}{\partial x_{j}}+u_{j}^{<} \frac{\partial \phi}{\partial x_{j}}
$$

or equivalently

$$
\frac{\mathcal{D} \phi}{\mathcal{D} t}=\frac{D \phi}{D t}+u_{j}^{<} \frac{\partial \phi}{\partial x_{j}}
$$

so that the averaging in the statistical sense of Eq. (147) is

$$
\left\langle\frac{\mathcal{D} \phi}{\mathcal{D} t}\right\rangle=\frac{\partial\langle\phi\rangle}{\partial t}+\left\langle u_{j}\right\rangle \frac{\partial\langle\phi\rangle}{\partial x_{j}}+\frac{\partial}{\partial x_{j}}\left\langle u_{j}^{<} \phi\right\rangle+u_{j}^{<} \frac{\partial \phi}{\partial x_{j}}
$$

On the other hand, applying a filtering process on Eq. (147) gives

$$
\frac{\overline{\mathcal{D} \phi}}{\mathcal{D} t}=\frac{\mathcal{D} \bar{\phi}}{\mathcal{D} t}
$$


Using (143), Eq. (149) can be rewritten as

$$
\left\langle\frac{\mathcal{D} \phi}{\mathcal{D} t}\right\rangle=\frac{\partial\langle\phi\rangle}{\partial t}+\left\langle u_{j}\right\rangle \frac{\partial\langle\phi\rangle}{\partial x_{j}}+\frac{\partial}{\partial x_{j}}\left\langle u_{j}^{<} \phi\right\rangle
$$

leading to

$$
\left\langle\frac{\mathcal{D} \phi}{\mathcal{D} t}\right\rangle=\frac{D\langle\phi\rangle}{D t}+\frac{\partial}{\partial x_{j}}\left\langle u_{j}^{<} \phi\right\rangle
$$

More generally, from the definition

$$
\frac{d \phi}{d t}=\frac{\partial \phi}{\partial t}+\left\langle u_{j}\right\rangle \frac{\partial \phi}{\partial x_{j}}+u_{j}^{<} \frac{\partial \phi}{\partial x_{j}}+u_{j}^{>} \frac{\partial \phi}{\partial x_{j}}
$$

and one can also easily derive some useful relations such as

$$
\begin{gathered}
\frac{\overline{d \phi}}{d t}=\frac{\mathcal{D} \bar{\phi}}{\mathcal{D} t}+\frac{\partial \overline{u_{j}^{>} \phi}}{\partial x_{j}} \\
\left\langle\frac{d \phi}{d t}\right\rangle=\frac{D\langle\phi\rangle}{D t}+\frac{\partial}{\partial x_{j}}\left\langle u_{j}^{<} \phi\right\rangle+\frac{\partial}{\partial x_{j}}\left\langle u_{j}^{>} \phi\right\rangle
\end{gathered}
$$

Finally, note that

$$
\frac{\partial}{\partial x_{j}}\left\langle u_{j}^{<\bar{\phi}}\right\rangle=\frac{\partial}{\partial x_{j}}\left\langle u_{j}^{<} \phi\right\rangle
$$

because $\left\langle u_{i}^{<} \phi^{>}\right\rangle$reduces to zero.

\section{Remark on turbulent flows near energetic equilibrium}

In the framework of first-moment closure referring to eddy-viscosity turbulence models, the production term, without prejudice to the reasoning, can be expressed as

$$
P^{(s)}=2 C_{\mu} \frac{k^{(s)^{2}}}{\varepsilon^{(s)}}\left(\bar{S}_{i j} \bar{S}_{i j}\right)
$$

with

$$
S_{i j}=\frac{1}{2}\left(\frac{\partial u_{i}}{\partial x_{j}}+\frac{\partial u_{j}}{\partial x_{i}}\right)
$$

and

$$
\nu_{t}^{(s)}=C_{\mu} \frac{k^{(s)^{2}}}{\varepsilon^{(s)}}
$$


so that

$$
\begin{aligned}
& \left\langle P^{(s)}\right\rangle \approx 2\left\langle\nu_{t}^{(s)}\right\rangle\left\langle S_{i j} S_{i j}\right\rangle \approx 2\left\langle\nu_{t}^{(s)}\right\rangle\left\langle S_{i j}\right\rangle\left\langle S_{i j}\right\rangle+2\left\langle\nu_{t}^{(s)}\right\rangle\left\langle S_{i j}^{<} S_{i j}^{<}\right\rangle \\
\approx & 2\left\langle\nu_{t}^{(s)}\right\rangle\left\langle S_{i j}\right\rangle\left\langle S_{i j}\right\rangle+\frac{1}{2}\left\langle\nu_{t}^{(s)}\right\rangle\left\langle\left(\frac{\partial u_{i}^{<}}{\partial x_{j}}+\frac{\partial u_{j}^{<}}{\partial x_{i}}\right)\left(\frac{\partial u_{i}^{<}}{\partial x_{j}}+\frac{\partial u_{j}^{<}}{\partial x_{i}}\right)\right\rangle
\end{aligned}
$$

where the last term is the mean spectral flux $F^{(1)}$. As it is clear from equation (160), the averaging in a statistical sense of the subfilter production (including the mean flow production and the production from the large resolved scales giving rise to $F^{(1)}$ approaches the RANS production, i.e., $\left\langle P^{(s)}\right\rangle \approx P$ and the same result prevails for the subfilter dissipation-rate $\left\langle\varepsilon^{(s)}\right\rangle \approx \epsilon$ for flows close to energetic equilibrium so that

$$
P \approx\left\langle P^{(s)}\right\rangle \approx 2 \nu_{t}\left\langle S_{i j}\right\rangle\left\langle S_{i j}\right\rangle
$$

where $\nu_{t}$ denotes the turbulent eddy viscosity in RANS modeling given by

$$
\nu_{t}=C_{\mu} \frac{k^{2}}{\varepsilon}
$$

involving the total kinetic energy. All these relations can be also rewritten

$$
\begin{array}{r}
P \approx 2\left\langle\nu_{t}^{(s)}\right\rangle\left\langle S_{i j}\right\rangle\left\langle S_{i j}\right\rangle+2\left\langle\nu_{t}^{(s)}\right\rangle\left\langle S_{i j}^{<} S_{i j}^{<}\right\rangle \\
\approx 2\left\langle\nu_{t}^{(s)}\right\rangle\left\langle\bar{S}_{i j} \bar{S}_{i j}\right\rangle \approx 2 \nu_{t}\left\langle S_{i j}\right\rangle\left\langle S_{i j}\right\rangle
\end{array}
$$

These relations are recalling the Heisenberg hypothesis is spectral space [55] assuming that the effect of the small eddies with wavenumbers larger than $\kappa$ by which energy is withdrawn from larger eddies with wavenumbers smaller than $\kappa$ is described through a small scales eddy viscosity. Then the spectral flux is represented as the product of two integrals in the domains $[0, \kappa]$ and $[\kappa, \infty]$ as in Eq. (163).

\section{Order of magnitude of the resolved part of the turbulent diffusion}

We consider the resolved part of the turbulent advection denoted $J^{(r)}$ of any arbitrary variable $\psi$ due to the macro-scale fluctuation velocity $u_{i}^{<}$as

$$
J_{\psi}^{(r)}(\psi)=-\frac{\partial}{\partial x_{j}}\left(u_{j}^{<} \psi^{<}\right)
$$


and which becomes a turbulent diffusion term once statistically averaged.

$$
\left\langle J_{\psi}^{(r)}(\psi)\right\rangle=-\frac{\partial}{\partial x_{j}}\left\langle u_{j}^{<} \psi^{<}\right\rangle
$$

The large scale fluctuation variable $\psi^{<}=\bar{\psi}-\langle\psi\rangle$ is fully resolved in the numerical simulation but for convenience an analytical approximation can be easily obtained considering the convection process by means of the large scale fluctuating velocity $u_{i}^{<}$and using the time scale of the resolved eddies $\tau^{(r)}$ as

$$
\psi^{<} \approx\langle\psi\rangle\left(x_{i}+u_{i}^{<} \tau^{(r)}\right)-\langle\psi\rangle\left(x_{i}\right)
$$

where

$$
\tau^{(r)} \approx \frac{k^{(r)}}{\epsilon^{(s)}}
$$

corresponds to the displacement time of an eddy before loosing its individuality. The approximation of the fluctuation corresponding to a wavelength range $\left[\kappa_{1}, \kappa_{2}\right]$ is approached by characteristic the time scale of this range of eddies multiplied by the gradient of the filtered field with a cutoff at $\kappa_{1}$. If $\kappa_{1}=0$, then the use of the gradient of the mean value is natural. Then, Taylor series expansion of Eq. (166) limited to the first order yields

$$
\psi^{<} \approx \tau^{(r)} u_{m}^{<} \frac{\partial\langle\psi\rangle}{\partial x_{m}}
$$

So that

$$
J_{\psi}^{(r)}=\frac{\partial}{\partial x_{j}}\left[u_{j}^{<} u_{m}^{<} \tau^{(r)} \frac{\partial\langle\psi\rangle}{\partial x_{m}}\right]
$$

or in a first approximation by neglecting the cross-correlations

$$
J_{\psi}^{(r)}(\psi)=-\frac{\partial}{\partial x_{j}} u_{j}^{<} \psi^{<} \approx \frac{\partial}{\partial x_{j}}\left[\frac{2}{3} u_{m}^{<} u_{m}^{<} \tau^{(r)} \frac{\partial\langle\psi\rangle}{\partial x_{j}}\right] \approx C_{\psi} \frac{\partial}{\partial x_{j}}\left[\frac{\left(k^{(r)}\right)^{2}}{\epsilon^{(s)}} \frac{\partial\langle\psi\rangle}{\partial x_{j}}\right]
$$

where $C_{\psi}$ is a numerical coefficient introduced to describe the diffusion process, leading to

$$
\left\langle J_{\psi}^{(r)}\right\rangle \approx C_{\psi} \frac{\partial}{\partial x_{j}}\left[\left\langle\frac{\left(k^{(r)}\right)^{2}}{\epsilon^{(s)}}\right\rangle \frac{\partial\langle\psi\rangle}{\partial x_{j}}\right]
$$

Moreover, for the instantaneous value $\bar{\psi}=\langle\psi\rangle+\psi^{<}$, a fluctuating part remains

$$
J_{\psi}^{(r)}(\psi)=\left\langle J_{\psi}^{(r)}\right\rangle+J_{\psi}^{(r)}<=C_{\psi} \frac{\partial}{\partial x_{j}}\left[\left\langle\frac{\left(k^{(r)}\right)^{2}}{\epsilon^{(s)}}\right\rangle \frac{\partial\langle\psi\rangle}{\partial x_{j}}\right]+J_{\psi}^{(r)}<
$$


Similarly, the diffusion process caused by the micro-scale fluctuation $\psi^{>}$is then can be computed as

$$
\psi^{>} \approx \bar{\psi}\left(x_{i}+u_{i}^{>} \tau^{(s)}\right)-\bar{\psi}\left(x_{i}\right)
$$

with

$$
\tau^{(s)} \approx \frac{k^{(s)}}{\epsilon^{(s)}}
$$

leading to

$$
\psi^{>} \approx \tau^{(s)} u_{m}^{>} \frac{\partial \bar{\psi}}{\partial x_{m}}
$$

so that

$$
J_{\psi}^{(s)}(\psi)=-\frac{\partial}{\partial x_{j}}\left(\overline{u_{j}^{>} \psi^{>}}\right)=C_{\psi} \frac{\partial}{\partial x_{j}}\left[\frac{\left(k^{(s)}\right)^{2}}{\epsilon^{(s)}} \frac{\partial \bar{\psi}}{\partial x_{j}}\right]=C_{\psi} \frac{\partial}{\partial x_{j}}\left[\frac{\left(k^{(s)}\right)^{2}}{\epsilon^{(s)}} \frac{\partial\langle\psi\rangle}{\partial x_{j}}\right]+C_{\psi} \frac{\partial}{\partial x_{j}}\left[\frac{\left(k^{(s)}\right)^{2}}{\epsilon^{(s)}} \frac{\partial \psi^{<}}{\partial x_{j}}\right]
$$

The total mean diffusion involving the micro-scale and the macro-scale is therefore

$$
\left\langle J_{\psi}^{(s+r)}\right\rangle=\left\langle J_{\psi}^{(s)}\right\rangle+\left\langle J_{\psi}^{(r)}\right\rangle=C_{\psi} \frac{\partial}{\partial x_{j}}\left[\left\langle\frac{\left(k^{(s)}\right)^{2}+\left(k^{(r)}\right)^{2}}{\epsilon^{(s)}}\right\rangle \frac{\partial\langle\psi\rangle}{\partial x_{j}}\right] \approx C_{\psi} \frac{\partial}{\partial x_{j}}\left[\left\langle\frac{\left(k^{(s)}+k^{(r)}\right)^{2}}{\epsilon^{(s)}}\right\rangle \frac{\partial\langle\psi\rangle}{\partial x_{j}}\right]
$$

or in a more compact form

$$
\left\langle J_{\psi}^{(s+r)}\right\rangle \approx C_{\psi} \frac{\partial}{\partial x_{j}}\left[\left\langle\frac{\left(k^{(s+r)}\right)^{2}}{\epsilon^{(s)}}\right\rangle \frac{\partial\langle\psi\rangle}{\partial x_{j}}\right]
$$

that is to say

$$
\left\langle J_{\psi}^{(s+r)}\right\rangle \approx C_{\psi} \frac{\partial}{\partial x_{j}}\left[\frac{k^{2}}{\epsilon} \frac{\partial\langle\bar{\psi}\rangle}{\partial x_{j}}\right]
$$

Note that the mean diffusion originating from the resolved scales is non zero only in the case of triple correlations. Indeed, as already remarked, double correlations between large scale and small scale fluctuations are always zero $\left\langle u_{i}^{<} u_{i}^{>}\right\rangle=0$. In contrast, for triple correlations like $\left\langle u_{j}^{<} k^{(s)^{\prime}}\right\rangle$ with $k^{(s)^{\prime}}=$ $k^{(s)<}+k^{(s)>}, k^{(s)<}=u_{i}^{<} u_{i}^{<}-\left\langle u_{i}^{<} u_{i}^{<}\right\rangle$and $k^{(s)>}=u_{i}^{>} u_{i}^{>}-\left\langle u_{i}^{>} u_{i}^{>}\right\rangle$, it appears $\left\langle u_{j}^{<} k^{(s)^{\prime}}\right\rangle=\left\langle u_{j}^{<} k^{(s)<}\right\rangle$. In practice, an approximation of $\left\langle J_{\psi}^{(s)}\right\rangle$ and $\left\langle J_{\psi}^{(r)}\right\rangle$ for practical use are thus

$$
\left\langle J_{\psi}^{(s)}\right\rangle=C_{\psi} \frac{\partial}{\partial x_{j}}\left[\frac{\left\langle k^{(s)}\right\rangle^{2}}{\left\langle\epsilon^{(s)}\right\rangle} \frac{\partial\langle\bar{\psi}\rangle}{\partial x_{j}}\right]
$$


and

$$
\left\langle J_{\psi}^{(r)}\right\rangle=C_{\psi} \frac{\partial}{\partial x_{j}}\left[\frac{\left\langle k^{(r)}\right\rangle^{2}}{\left\langle\epsilon^{(s)}\right\rangle} \frac{\partial\langle\bar{\psi}\rangle}{\partial x_{j}}\right]
$$

We have thus approximated both the large scale fluctuating diffusion term and the small scale averaged one in a consistent way.

\section{E Variational calculus of the diffusion terms $J_{\psi}^{(s+r)}(\psi)$}

The general expressions of the diffusion terms $J_{\psi}^{(r)}(\psi)$ and $J_{\psi}^{(s)}(\psi)$ are given by Eqs. (172) and (176) in which $k^{(r)}, k^{(s)}$ and $\epsilon^{(s)}$ are all functions of $\left(x_{i}, t\right)$. In a first approximation, the functional variations of $\psi\left(x_{i}, t\right)$ can be computed as $\delta \psi\left(x_{i}, t\right)=\zeta\left(x_{i}, t\right) \delta \alpha$, where $\zeta\left(x_{i}, t\right)$ is a function in space and time while $\delta \alpha$ is a small scalar increment, it clearly emphasizes the fact that $\delta$ is a functional variation of $\psi$ whose magnitude is controlled by $\delta \alpha$ while keeping the same shape $\zeta\left(x_{i}, t\right)$. If $\partial / \partial x_{i}$ is the usual partial differential operator in $x_{i}$ (or $t$ ) corresponding to the usual first partial derivative, then one can write

$$
\delta\left(\frac{\partial \psi}{\partial x_{i}}\right)\left(x_{i}, t\right)=\frac{\partial(\delta \psi)}{\partial x_{i}}\left(x_{i}, t\right)=\frac{\partial}{\partial x_{i}}\left[\zeta\left(x_{i}, t\right)\right] \delta \alpha
$$

regardless the particular shape of the $\psi\left(x_{i}, t\right)$ curve. Then, assuming that the function $\zeta\left(x_{i}, t\right)$ roughly keeps the same shape as the original function $\psi\left(x_{i}, t\right)$, that is to say

$$
\zeta\left(x_{i}, t\right) \propto \psi\left(x_{i}, t\right)
$$

we get the following estimate

$$
\frac{\delta\left(\frac{\partial \psi}{\partial x_{i}}\right)}{\frac{\partial \psi}{\partial x_{i}}}\left(x_{i}, t\right)=\frac{\delta \psi}{\psi}\left(x_{i}, t\right)=\delta \alpha
$$

Applied to Eq. (178), one gets

$$
\frac{\delta\left\langle J_{\psi}^{(s+r)}\right\rangle}{\left\langle J_{\psi}^{(s+r)}\right\rangle}\left(x_{i}, t\right) \approx \frac{\delta\left[\left\langle\frac{\left(k^{(s+r)}\right)^{2}}{\epsilon^{(s)}}\right\rangle \frac{\partial\langle\psi\rangle}{\partial x_{j}}\right]}{\left[\left\langle\frac{\left(k^{(s+r)}\right)^{2}}{\epsilon^{(s)}}\right\rangle \frac{\partial\langle\psi\rangle}{\partial x_{j}}\right]}\left(x_{i}, t\right)
$$

and thus

$$
\frac{\delta\left\langle J_{\psi}^{(s+r)}\right\rangle}{\left\langle J_{\psi}^{(s+r)}\right\rangle}\left(x_{i}, t\right) \approx 2 \frac{\delta\left\langle k^{(s+r)}\right\rangle}{\left\langle k^{(s+r)}\right\rangle}\left(x_{i}, t\right)-\frac{\delta\left\langle\epsilon^{(s)}\right\rangle}{\left\langle\epsilon^{(s)}\right\rangle}\left(x_{i}, t\right)+\frac{\delta\langle\psi\rangle}{\langle\psi\rangle}\left(x_{i}, t\right)
$$


for $\psi=k^{(s)}\left(x_{i}, t\right)$ and $\psi=\epsilon^{(s)}\left(x_{i}, t\right)$, respectively, considering that $k^{(s+r)}=k^{(s)}+k^{(r)}$ remains approximatively constant when the cutoff is varied, Eq. (186) reduces to

$$
\frac{\delta\left\langle J_{\psi}^{(s+r)}\right\rangle}{\left\langle J_{\psi}^{(s+r)}\right\rangle}\left(x_{i}, t\right)=-\frac{\delta\left\langle\epsilon^{(s)}\right\rangle}{\left\langle\epsilon^{(s)}\right\rangle}\left(x_{i}, t\right)+\frac{\delta\langle\psi\rangle}{\langle\psi\rangle}\left(x_{i}, t\right)
$$

Due to the implicit $j$ summation in Eq. (178), one may reasonably wonder if Eq. (185) is valid. This equation is still verified because for each value of $j,(j=1,2$ and 3$)$, the same approximation given by Eq. (186) is obtained so that

$$
\frac{\delta\left\langle J_{\psi}^{(s+r)}\right\rangle}{\left\langle J_{\psi}^{(s+r)}\right\rangle}=\frac{\delta\left\langle J_{\psi, j=1}^{(s+r)}\right\rangle}{\left\langle J_{\psi, j=1}^{(s+r)}\right\rangle}=\frac{\delta\left\langle J_{\psi, j=2}^{(s+r)}\right\rangle}{\left\langle J_{\psi, j=2}^{(s+r)}\right\rangle}=\frac{\delta\left\langle J_{\psi, j=3}^{(s+r)}\right\rangle}{\left\langle J_{\psi, j=3}^{(s+r)}\right\rangle}=\frac{\delta\left\langle J_{\psi, j=1,3}^{(s+r)}\right\rangle}{\left\langle J_{\psi, j=1,3}^{(s+r)}\right\rangle}
$$

Note that the relations (182) and (184) are also valid for instantaneous quantities but in this case $\delta \alpha$ becomes fluctuating.

\section{Compliance with Ethical Standards}

Conflict of interest The authors declare that they have no conflict of interest.

\section{References}

[1] Spalart, P.R.: Strategies for turbulence modelling and simulations. Int. J. Heat Fluid Flow 21, $252-263(2000)$

[2] Fröhlich J., Von Terzi, D.: Hybrid LES/RANS methods for the simulation of turbulent flows. Prog. Aerosp. Sci. 44, 349-377 (2008)

[3] Chaouat, B.: The state of the art of hybrid RANS/LES modeling for the simulation of turbulent flows. Flow, Turbul. Combust. 99, 279-327 (2017)

[4] Durbin, P..: Some Recent Developments in Turbulence Closure Modeling. Annu. Rev. Fluid Mech. 50, 77-103 (2017) 
[5] Heinz, S.: A review of hybrid RANS-LES methods for turbulent flows: Concepts and applications. Prog. Aerosp. Sci. 114, (100595), 1-25 (2020)

[6] Fasel, H.F., Seidel, J., Wernz, S.: A methodology for simulations of complex turbulent flows. J. Fluids Engng. ASME 124(4), 933-942 (2002)

[7] Speziale, C.G.: Turbulence modeling for time-dependent RANS and VLES : A review. AIAA J. 36, 173-184 (1998)

[8] Spalart, P.R.: Detached-eddy simulation. Annu. Rev. Fluid Mech. 41,181-202 (2009)

[9] Shur, M.L., Spalart, P.R., Strelets, M.K., Travin, K.: A hybrid RANS-LES approach with delayedDES and wall-modelled LES capabilities. Int. J. Heat Fluid Flow 29, 1638-1649 (2008)

[10] Schiestel, R., Dejoan, A.: Towards a new partially integrated transport model for coarse grid and unsteady turbulent flow simulations. Theoret. Comput. Fluid Dyn. 18, 443-468 (2005)

[11] Chaouat, B., Schiestel, R.: A new partially integrated transport model for subgrid-scale stresses and dissipation rate for turbulent developing flows. Phys. Fluids 17, 065106, 1-19 (2005)

[12] Girimaji, S.S., Abdol-Hamid, K.S.: Partially-averaged Navier Stokes Model for Turbulence: Implementation and Validation. AIAA-paper $n^{\circ}$ 0502, 1-14 (2005)

[13] Girimaji, S.S.: Partially-averaged Navier-Stokes method for turbulence: A Reynolds averaged Navier-Stokes to direct numerical simulation bridging method. ASME J. Appl. Mech. 73(3), 413$421(2006)$

[14] Girimaji, S.S., Jeong, E., Srinivasan, R.: Partially averaged Navier-Stokes method for turbulence : Fixed point analysis and comparisons with unsteady partially averaged Navier-Stokes. ASME J. Appl. Mech. 73(3), 422-429 (2006)

[15] Menter, F.R., Egorov, Y.: The scale-adaptive simulation method for unsteady turbulent flow prediction : Part 1 : Theory and model description. Flow, Turbul. Combust. 85, 113-138 (2020) 
[16] Egorov, Y., Menter, F.T., Lechner R., Cokljat, D.: The scale-adaptive simulation method for unsteady turbulent flow prediction : Part 2 : Application to complex flows. Flow, Turbul. Combust. 85, 139-165 (2020)

[17] Jeandel D., Brison, J.F., Mathieu, J.: Modeling methods in physical and spectral spaces. Phys. Fluids. 100, 21, 169-181 (1978)

[18] Cambon, C., Jeandel, D., Mathieu, J.: Spectral modelling of homogeneous non-isotropic turbulence. J. Fluid Mech. 104, 247-262 (1981)

[19] Mons, V., Cambon, Sagaut, P.: A spectral model for homogeneous shear-driven anisotropic turbulence in terms of spherically averaged descriptors. J. Fluid Mech. 788, 147-182 (2016)

[20] Chaouat, B., Schiestel, R.: Progress in subgrid-scale transport modelling for continuous hybrid non-zonal RANS/LES simulations. Int. J. Heat Fluid Flow 30, 602-616 (2009)

[21] Chaouat, B., Schiestel, R.: Extension of the partially integrated transport modeling method to the simulation of passive scalar turbulent fluctuations at various Prandtl numbers. Submitted to Int. J. Heat Fluid Flow (2020)

[22] Chaouat B., Schiestel, R.: From single-scale turbulence models to multiple-scale and subgrid-scale models by Fourier transform. Theoret. Comput. Fluid Dyn. 21, pp 201-229 (2007)

[23] Chaouat, B., Schiestel, R.: Hybrid RANS-LES simulations of the turbulent flow over periodic hills at high Reynolds number using the PITM method. Comput. Fluids 84, 279-300 (2013)

[24] Razi, P., Tazraei, P., Girimaj, S.S.: Partially-averaged Navier-Stokes (PANS) simulations of flow separation over smooth curved surfaces. Int. J. Heat Fluid Flow. 21, 252-263 (2017)

[25] Foroutan, H., Yavuzkurt, S.: A partially averaged Navier Stokes model for the simulation of turbulent swirling flow with vortex breakdown. Int. J. Heat Fluid Flow 50, 402-416 (2014)

[26] Davidson, L., Friess, C.: A new formulation of $f_{k}$ for the PANS model. J. Turbul. 20(5), 322-336 (2019) 
[27] Klapwijk, M., Lloyd, T., Vaz, G.: On the accuracy of partially averaged Navier-Stokes resolution estimates. Int. J. Heat Fluid Flow 80, 108484, 1-10 (2019)

[28] Tazraei, P., Girimaji, S.S.: Scale-resolving simulations of turbulence: Equilibrium boundary layer analysis leading to near-wall closure modeling. Phys. Rev. Fluids 4, 104607, 1-17 (2019)

[29] Kamble, C., Girimaji, S.S., Chen, H.: Partially averaged Navier-Stokes formulation of a two-layer turbulence model. AIAA J. 58(1), 174-183 (2020)

[30] Chaouat, B.: Subfilter scale transport model for hybrid RANS/LES simulations applied to a complex bounded flow. J. Turbul. 11(51), 1-30 (2010)

[31] Heinz, S., Mokhtarpoor, R., Stoellinger, M.: Physics-Based Control of Hybrid RANS-LES Methods. AIAA-paper $n^{\circ} \mathbf{0 3 2 7}, 1-12(2019)$

[32] Heinz, S.: The large eddy simulation capability of Reynolds-averaged Navier-Stokes equations: Analytical results. Phys. Fluids 31, 021702, 1-6 (2019)

[33] Heinz, S., Mokhtarpoor R., Stoellinger M.: Theory-based Reynolds-averaged Navier-Stokes equations with large eddy simulation capability for separated turbulent flow simulations. Phys. Fluids 32, 065102, 1-20 (2020)

[34] Jakirlic, S., Maduta, R.: Extending the bounds of steady RANS closures: Toward an instabilitysensitive Reynolds stress model. Int. J. Heat Fluid Flow 51, 175-194 (2015)

[35] Chaouat, B.: Commutation errors in PITM simulations. Int. J. Heat Fluid Flow 67, 138-154 (2017)

[36] Chaouat, B., Schiestel, R.: Partially integrated transport modeling method for turbulence simulation with variable filters. Phys. Fluids 25, 125102, 1-39 (2013)

[37] Germano, M.: Turbulence : the filtering approach. J. Fluid Mech. 41, 238, 325-336 (1992)

[38] Schiestel, R.: Modeling and simulation of turbulent flows. ISTE Ltd and J. Wiley (2008)

[39] Hamba, F.: Log-layer mismatch and commutation error in hybrid RANS/LES simulation of channel flow. Int. J. Heat Fluid Flow 30, 20-31 (2009) 
[40] Friess, C., Davidson, L.: A formulation of PANS capable of mimicking IDDES. Int. J. Heat Fluid Flow 86, 108666, 1-25 (2020)

[41] Deardorff, J.W.: A numerical study of three-dimensional turbulent channel flow at large Reynolds numbers. J. Fluid Mech. 41, 453-480 (1970)

[42] Bardina, J., Ferziger, J.H., Reynolds, W.C: Improved subgrid scale models for large eddy simulations. AIAA-paper $n^{\circ} \mathbf{1 3 5 7}, 1-10(1980)$

[43] Chaouat, B.: Simulation of turbulent rotating flows using a subfilter scale stress model derived from the partially integrated transport modeling method. Phys. Fluids 24, 045108, 1-35 (2012)

[44] Scotti, A., Meneveau, Ch.: Generalized Smagorinsky model for anisotropic grids. Phys. of Fluids A, 5(9), 2306-2308 (1993)

[45] Scotti, A., Meneveau, Ch., Fatica, M.: Dynamic Smagorinsky model on anisotropic grids. Phys. of Fluids 9(6), 1856-1858 (1997)

[46] Chaouat, B., Peyret, C.: Investigation of the wall scalar fluctuations effect on passive scalar turbulent fields at several Prandtl numbers by means of direct numerical simulations. J. Heat Transfer, ASME 141, 1-9. (2019)

[47] Chaouat, B.: An efficient numerical method for RANS/LES turbulent simulations using subfilter scale stress transport equations. Int. J. Numer. Methods Fluids 67, 1207-1233 (2011)

[48] Yassour, Y., Wolfshtein, M.: A spectral model for the calculation of homogeneous turbulent flows. Symposium on Turbulent Shear Flows IV, Karlsruhe Univ. 9.23-9.29, (1983)

[49] Chaouat, B., Schiestel, R.: Analytical insights into the partially integrated transport modeling method for hybrid Reynolds averaged Navier-Stokes equations-large eddy simulations of turbulent flows. Phys. Fluids 24, 085106, 1-34 (2012)

[50] Vichnevetsky, R.: Fourier analysis of numerical approximation of hyperbolic equations. SIAM, Philadelphia (1982) 
[51] Press W. H., Teukolsky S. A, Vetterling W. T. and Flannery B. P.: Numerical recipes. Cambridge University Press (1992)

[52] Befeno, I., Schiestel, R.: Non-equilibrium mixing of turbulence scales using a continuous hybrid RANS/LES approach: Application to the shearless mixing layer. Flow, Turbul. Combust. 78, 129-151 (2007)

[53] Chaouat, B.: Application of the PITM method using inlet synthetic turbulence generation for the simulation of the turbulent flow in a small axisymmetric contraction. Flow, Turbul. Combust. 98, 987-1024 (2017)

[54] Chaouat, B., Schiestel, R.: Simulations of turbulent flows out of spectral equilibrium using the PITM method, 23ème Congrès Français de Mécanique, 2491-715X, 1-16 (2017)

[55] Hinze, J.O.: Turbulence, (Mc Graw-Hill, New York), (1975) 